\title{
Search for charged lepton flavor violation of vector mesons in the BLMSSM model
}

\author{
Xing-Xing Dong, ${ }^{1, *}$ Shu-Min Zhao, ${ }^{1, \dagger}$ Jing-Jing Feng, ${ }^{1}$ Guo-Zhu Ning, ${ }^{1, \star}$ \\ Jian-Bin Chen, ${ }^{2,8}$ Hai-Bin Zhang, ${ }^{1}$ and Tai-Fu Feng ${ }^{1}$ \\ ${ }^{1}$ Department of Physics and Technology, Hebei University, Baoding 071002, China \\ ${ }^{2}$ Department of Physics, Taiyuan University of Technology, Taiyuan 030024, China
}

(Received 25 December 2017; revised manuscript received 7 March 2018; published 30 March 2018)

\begin{abstract}
We analyze the charged lepton flavor violating (CLFV) decays of vector mesons $V \rightarrow l_{i}^{ \pm} l_{j}^{\mp}$ with $V \in$ $\left\{\phi, J / \Psi, \Upsilon, \rho^{0}, \omega\right\}$ in the BLMSSM model. This new model is introduced as a supersymmetric extension of the Standard Model (SM), where local gauged baryon number $B$ and lepton number $L$ are considered. The numerical results indicate the BLMSSM model can produce significant contributions to such two-body CLFV decays, and the branching ratios to these CLFV processes can easily reach the present experimental upper bounds. Therefore, searching for CLFV processes of vector mesons may be an effective channel to study new physics.
\end{abstract}

DOI: 10.1103/PhysRevD.97.056027

\section{INTRODUCTION}

The neutrino oscillation experiments have convinced us that neutrinos possess tiny masses and mix with each other [1-9]. This phenomenon shows that the charged lepton flavor violating (CLFV) process [10-12] is strongly suppressed in the standard model (SM). Therefore, CLFV processes are considered as evidence for the study of new physics beyond the SM. Physicists now do more research on the CLFV decays of vector mesons in various SM extensions, such as grand unified models [13,14], supersymmetric models with and without R-party [15-17], leftright symmetry models [18,19], and Z' models [20,21], etc. In our previous work, we investigate these CLFV processes in the framework of MSSM with a type-I see-saw mechanism[22,23], and some of the theoretical evaluations on CLFV processes fit better with the experimental upper bounds, such as $J / \Psi(\Upsilon) \rightarrow l_{i}^{ \pm} l_{j}^{\mp}$ with $l_{i}, l_{j} \in\{e, \mu, \tau\}$. However, the predictions on processes $\phi\left(\rho^{0}, \omega\right) \rightarrow e^{+} \mu^{-}$in the MSSM with the type-I seesaw mechanism are around $10^{-20}$, which are far below the present experimental upper bounds.

\footnotetext{
*dxx_0304@163.com

†zhaosm@hbu.edu.cn

*nkninggz@163.com

j.b.chen1007@gmail.com
}

Published by the American Physical Society under the terms of the Creative Commons Attribution 4.0 International license. Further distribution of this work must maintain attribution to the author(s) and the published article's title, journal citation, and DOI. Funded by SCOAP.
Current experiments naturally suggest searching for twobody decays of vector mesons in the $e^{ \pm} \mu^{\mp}$ final state. Considering the experimental constraints on the processes $l_{j} \rightarrow 3 l_{i}$ and $l_{j} \rightarrow l_{i}+\gamma$, many experts have studied the CLFV processes involving vector mesons $V \rightarrow l_{i}^{ \pm} l_{j}^{\mp}$ with $V \in\left\{\phi, J / \Psi, \Upsilon, \rho^{0}, \omega\right\}$ and $l_{i}, l_{j} \in\{e, \mu, \tau\} \quad[24,25]$. Likewise, the constraints on the $\mu-e$ conversion are also taken into account on these processes [26-28]. Currently, PDG [29] give independent experimental upper limits of the two-body decays of vector mesons, which are shown as

$$
\begin{aligned}
\operatorname{Br}\left(\phi \rightarrow e^{+} \mu^{-}\right) & \leq 2.0 \times 10^{-6} \\
\operatorname{Br}\left(J / \Psi \rightarrow e^{+} \mu^{-}\right) & \leq 1.6 \times 10^{-7} \\
\operatorname{Br}\left(J / \Psi \rightarrow e^{+} \tau^{-}\right) & \leq 8.3 \times 10^{-6} \\
\operatorname{Br}\left(J / \Psi \rightarrow \mu^{+} \tau^{-}\right) & \leq 2.0 \times 10^{-6} \\
\operatorname{Br}\left(\Upsilon \rightarrow \mu^{+} \tau^{-}\right) & \leq 6.0 \times 10^{-6}
\end{aligned}
$$

As an extension of the minimal supersymmetric standard model (MSSM)[15,16,30,31], the gauged baryon number $(B)$ and lepton number $(L)$ are added in the BLMSSM [32-35]. Compared with the MSSM possessing R-parity conservation, there are new parameters and new contributions to CLFV processes. We introduce the local gauged $B$ to explain the matter-antimatter asymmetry in the universe. Lepton number $L$ is expected to be broken spontaneously at the $\mathrm{TeV}$ scale. Furthermore, right-handed neutrinos are considered to explain the neutrino oscillation experiments. In our work, the CLFV processes of vector mesons $\left(\phi\left(J / \Psi, \Upsilon, \rho^{0}, \omega\right) \rightarrow e^{+} \mu^{-}\right.$and $\left.J / \Psi(\Upsilon) \rightarrow e^{+} \tau^{-}\left(\mu^{+} \tau^{-}\right)\right)$ are investigated within the BLMSSM. Let us assume that 
a vector meson $V_{i}$ couples to $e^{ \pm} \mu^{\mp}$, and the corresponding effective Lagrangian can be written as [25-28]

$$
\mathcal{L}_{\text {eff }}=V_{i}^{\nu}\left(\xi_{V}^{M} \bar{\mu} \gamma_{\nu} e+\xi_{A}^{M} \bar{\mu} \gamma_{\nu} \gamma_{5} e+\text { H.c. }\right),
$$

where $V_{i}$ is a quark-antiquark bound state like $\phi, J /$ $\Psi, \Upsilon, \rho^{0}, \omega . \xi_{V, A}^{M}$ represent the effective couplings of the vector meson $V_{i}$ to the CLFV lepton currents.

This work is organized as follows: In Sec. II, we describe the BLMSSM model briefly, including the corresponding interaction Lagrangian, needed mass matrices, and couplings. We derive the analytic results of amplitudes for diagrams in Sec. III. In Sec. IV, we give the corresponding parameters and numerical results, and the conclusion is shown in Sec. V. In the Appendix, we discuss the corresponding hadron matrix elements, one-loop integral functions, and the superfields in the BLMSSM.

\section{BLMSSM}

In this work, we study a supersymmetric model where baryon $(B)$ and lepton $(L)$ numbers are local gauge symmetries. This model is defined as the BLMSSM and the corresponding local gauge group is $S U(3)_{C} \otimes S U(2)_{L} \otimes$ $U(1)_{Y} \otimes U(1)_{B} \otimes U(1)_{L}[32,36,37]$. In the BLMSSM, the local $B$ and $L$ are spontaneously broken at the TeV scale. In order to cancel the $B$ and $L$ anomalies, a vectorlike family is needed, which are $\hat{Q}_{4}, \hat{U}_{4}^{c}, \hat{D}_{4}^{c}, \hat{L}_{4}, \hat{E}_{4}^{c}, \hat{N}_{4}^{c}$ and $\hat{Q}_{5}^{c}, \hat{U}_{5}, \hat{D}_{5}, \hat{L}_{5}^{c}, \hat{E}_{5}, \hat{N}_{5}$. The corresponding superfields presented in the BLMSSM are shown in Table I of the Appendix. To break the baryon number and lepton number spontaneously, the model introduces the Higgs superfields $\hat{\Phi}_{B}, \hat{\varphi}_{B}$ and $\hat{\Phi}_{L} \hat{\varphi}_{L}$, respectively. After these Higgs superfields acquire nonzero vacuum expectation values (VEVs), the exotic quarks and leptons obtain masses. Furthermore, the introduction of superfields $\hat{X}$ and $\hat{X}^{\prime}$ is to make the exotic quarks avoid stability. Actually, the lightest one can be a dark matter candidate.

The superpotential of the BLMSSM is written as

$$
\mathcal{W}_{\text {BLMSSM }}=\mathcal{W}_{\text {MSSM }}+\mathcal{W}_{B}+\mathcal{W}_{L}+\mathcal{W}_{X}
$$

where $\mathcal{W}_{\text {MSSM }}$ represents the superpotential of the MSSM, and the concrete forms of superpotentials $\mathcal{W}_{B}, \mathcal{W}_{L}$, and $\mathcal{W}_{X}$ are given as

$$
\begin{aligned}
& \mathcal{W}_{B}=\lambda_{Q} \hat{Q}_{4} \hat{Q}_{5}^{c} \hat{\Phi}_{B}+\lambda_{U} \hat{U}_{4}^{c} \hat{U}_{5} \hat{\varphi}_{B}+\lambda_{D} \hat{D}_{4}^{c} \hat{D}_{5} \hat{\varphi}_{B}+\mu_{B} \hat{\Phi}_{B} \hat{\varphi}_{B}+Y_{u_{4}} \hat{Q}_{4} \hat{H}_{u} \hat{U}_{4}^{c}+Y_{d_{4}} \hat{Q}_{4} \hat{H}_{d} \hat{D}_{4}^{c}+Y_{u_{5}} \hat{Q}_{5}^{c} \hat{H}_{d} \hat{U}_{5}+Y_{d_{5}} \hat{Q}_{5}^{c} \hat{H}_{u} \hat{D}_{5}, \\
& \mathcal{W}_{L}=Y_{e_{4}} \hat{L}_{4} \hat{H}_{d} \hat{E}_{4}^{c}+Y_{\nu_{4}} \hat{L}_{4} \hat{H}_{u} \hat{N}_{4}^{c}+Y_{e_{5}} \hat{L}_{5}^{c} \hat{H}_{u} \hat{E}_{5}+Y_{\nu_{5}} \hat{L}_{5}^{c} \hat{H}_{d} \hat{N}_{5}+Y_{\nu} \hat{L} \hat{H}_{u} \hat{N}^{c}+\lambda_{N^{c}} \hat{N}^{c} \hat{N}^{c} \hat{\varphi}_{L}+\mu_{L} \hat{\Phi}_{L} \hat{\varphi}_{L} \\
& \mathcal{W}_{X}=\lambda_{1} \hat{Q} \hat{Q}_{5}^{c} \hat{X}+\lambda_{2} \hat{U}^{c} \hat{U}_{5} \hat{X}^{\prime}+\lambda_{3} \hat{D}^{c} \hat{D}_{5} \hat{X}^{\prime}+\mu_{X} \hat{X} \hat{X}^{\prime}
\end{aligned}
$$

The soft breaking terms $\mathcal{L}_{\text {soft }}$ in the BLMSSM can be found in our previous work [38-40].

In the BLMSSM, the $S U(2)_{L}$ doublets $H_{u}, H_{d}$ and singlets $\Phi_{B}, \varphi_{B}, \Phi_{L}, \varphi_{L}$ obtain the nonzero VEVs $v_{u}, v_{d}$ and $v_{B}, \bar{v}_{B}$, $v_{L}, \bar{v}_{L}$, respectively, then the local gauge symmetry $S U(2)_{L} \otimes U(1)_{Y} \otimes U(1)_{B} \otimes U(1)_{L}$ breaks down to the electromagnetic symmetry $U(1)_{e}$.

$$
\begin{aligned}
& H_{u}=\left(\begin{array}{c}
H_{u}^{+} \\
\frac{1}{\sqrt{2}}\left(v_{u}+H_{u}^{0}+i P_{u}^{0}\right)
\end{array}\right), \quad H_{d}=\left(\begin{array}{c}
\frac{1}{\sqrt{2}}\left(v_{d}+H_{d}^{0}+i P_{d}^{0}\right) \\
H_{d}^{-}
\end{array}\right), \quad \Phi_{B}=\frac{1}{\sqrt{2}}\left(v_{B}+\Phi_{B}^{0}+i P_{B}^{0}\right), \\
& \varphi_{B}=\frac{1}{\sqrt{2}}\left(\bar{v}_{B}+\varphi_{B}^{0}+i \bar{P}_{B}^{0}\right), \quad \Phi_{L}=\frac{1}{\sqrt{2}}\left(v_{L}+\Phi_{L}^{0}+i P_{L}^{0}\right), \quad \varphi_{L}=\frac{1}{\sqrt{2}}\left(\bar{v}_{L}+\varphi_{L}^{0}+i \bar{P}_{L}^{0}\right) .
\end{aligned}
$$

In this model, we introduce the superfields $\hat{N}^{c}$, so three neutrinos obtain tiny masses through the see-saw mechanism. In the basis $\left(\psi_{\nu_{L}^{I}}, \psi_{N_{R}^{c l}}\right)$, the mass matrix of neutrinos is deduced after the symmetry breaking,

$$
\begin{aligned}
Z_{N_{\nu}}^{\top}\left(\begin{array}{cc}
0 & \frac{v_{u}}{\sqrt{2}}\left(Y_{\nu}\right)^{I J} \\
\frac{v_{u}}{\sqrt{2}}\left(Y_{\nu}^{T}\right)^{I J} & \frac{\bar{v}_{L}}{\sqrt{2}}\left(\lambda_{N^{c}}\right)^{I J}
\end{array}\right) Z_{N_{\nu}}=\operatorname{diag}\left(m_{\nu^{\alpha}}\right), \quad \alpha=1 \ldots 6, I, J=1,2,3, \\
\psi_{\nu_{L}^{I}}=Z_{N_{\nu}}^{I \alpha} k_{N_{\alpha}}^{0}, \quad \psi_{N_{R}^{c l}}=Z_{N_{\nu}}^{(I+3) \alpha} k_{N_{\alpha}}^{0}, \quad \chi_{N_{\alpha}}^{0}=\left(\begin{array}{c}
k_{N_{\alpha}}^{0} \\
k_{N_{\alpha}}^{0}
\end{array}\right) .
\end{aligned}
$$

Here, $\chi_{N_{\alpha}}^{0}$ represent the mass eigenstates of the neutrino fields mixed by left-handed and right-handed neutrinos.

Similarly, with the introduced superfields $\hat{N}^{c}$, we can also obtain the mass squared matrix of sneutrinos in the base $\tilde{n}^{T}=\left(\tilde{\nu}, \tilde{N}^{c}\right)$, and this matrix is more complicated than that in the MSSM. 


$$
\left(\begin{array}{cc}
\mathcal{M}_{\tilde{n}}^{2}\left(\tilde{\nu}_{I}^{*} \tilde{\nu}_{J}\right) & \mathcal{M}_{\tilde{n}}^{2}\left(\tilde{\nu}_{I} \tilde{N}_{J}^{c}\right) \\
\left(\mathcal{M}_{\tilde{n}}^{2}\left(\tilde{\nu}_{I} \tilde{N}_{J}^{c}\right)\right)^{\dagger} & \mathcal{M}_{\tilde{n}}^{2}\left(\tilde{N}_{I}^{c *} \tilde{N}_{J}^{c}\right)
\end{array}\right)
$$

where

$$
\begin{aligned}
\mathcal{M}_{\tilde{n}}^{2}\left(\tilde{\nu}_{I}^{*} \tilde{\nu}_{J}\right) & =\frac{g_{1}^{2}+g_{2}^{2}}{8}\left(v_{d}^{2}-v_{u}^{2}\right) \delta_{I J}+g_{L}^{2}\left(\bar{v}_{L}^{2}-v_{L}^{2}\right) \delta_{I J}+\frac{v_{u}^{2}}{2}\left(Y_{\nu}^{\dagger} Y_{\nu}\right)_{I J}+\left(m_{\tilde{L}}^{2}\right)_{I J}, \\
\mathcal{M}_{\tilde{n}}^{2}\left(\tilde{N}_{I}^{c *} \tilde{N}_{J}^{c}\right) & =-g_{L}^{2}\left(\bar{v}_{L}^{2}-v_{L}^{2}\right) \delta_{I J}+\frac{v_{u}^{2}}{2}\left(Y_{\nu}^{\dagger} Y_{\nu}\right)_{I J}+2 \bar{v}_{L}^{2}\left(\lambda_{N^{c}}^{\dagger} \lambda_{N^{c}}\right)_{I J}+\left(m_{\tilde{N}^{c}}^{2}\right)_{I J}+\mu_{L} \frac{v_{L}}{\sqrt{2}}\left(\lambda_{N^{c}}\right)_{I J}-\frac{\bar{v}_{L}}{\sqrt{2}}\left(A_{N^{c}}\right)_{I J}\left(\lambda_{N^{c}}\right)_{I J}, \\
\mathcal{M}_{\tilde{n}}^{2}\left(\tilde{\nu}_{I} \tilde{N}_{J}^{c}\right) & =\mu^{*} \frac{v_{d}}{\sqrt{2}}\left(Y_{\nu}\right)_{I J}-v_{u} \bar{v}_{L}\left(Y_{\nu}^{\dagger} \lambda_{N^{c}}\right)_{I J}+\frac{v_{u}}{\sqrt{2}}\left(A_{N}\right)_{I J}\left(Y_{\nu}\right)_{I J} .
\end{aligned}
$$

Through matrix $Z_{\nu}$, the mass matrix can be diagonalized.

With the new gaugino $\lambda_{L}$ and the superpartners of $S U(2)_{L}$ singlets $\Phi_{L}, \varphi_{L}$ mixing together, the mass matrix of lepton neutralinos $\chi_{L}^{0}$ is produced. In the base $\left(i \lambda_{L}, \psi_{\Phi_{L}}, \psi_{\varphi_{L}}\right)$, we can diagonalize the mass matrix $\chi_{L}^{0}$ by $Z_{N_{L}}$.

$$
\mathcal{L}_{\chi_{L}^{0}}=\frac{1}{2}\left(i \lambda_{L}, \psi_{\Phi_{L}}, \psi_{\varphi_{L}}\right)\left(\begin{array}{ccc}
2 M_{L} & 2 v_{L} g_{L} & -2 \bar{v}_{L} g_{L} \\
2 v_{L} g_{L} & 0 & -\mu_{L} \\
-2 \bar{v}_{L} g_{L} & -\mu_{L} & 0
\end{array}\right)\left(\begin{array}{c}
i \lambda_{L} \\
\psi_{\Phi_{L}} \\
\psi_{\varphi_{L}}
\end{array}\right)+\text { H.c. }
$$

From the contributions of the superpotential and the soft breaking terms, the corrected form for the slepton mass squared matrix reads as

$$
\left(\begin{array}{ll}
\left(\mathcal{M}_{L}^{2}\right)_{L L} & \left(\mathcal{M}_{L}^{2}\right)_{L R} \\
\left(\mathcal{M}_{L}^{2}\right)_{L R}^{\dagger} & \left(\mathcal{M}_{L}^{2}\right)_{R R}
\end{array}\right)
$$

where

$$
\begin{aligned}
& \left(\mathcal{M}_{L}^{2}\right)_{L L}=\frac{\left(g_{1}^{2}-g_{2}^{2}\right)\left(v_{d}^{2}-v_{u}^{2}\right)}{8} \delta_{I J}+g_{L}^{2}\left(\bar{v}_{L}^{2}-v_{L}^{2}\right) \delta_{I J}+m_{l^{l}}^{2} \delta_{I J}+\left(m_{\tilde{L}}^{2}\right)_{I J}, \\
& \left(\mathcal{M}_{L}^{2}\right)_{L R}=\frac{\mu^{*} v_{u}}{\sqrt{2}}\left(Y_{l}\right)_{I J}-\frac{v_{u}}{\sqrt{2}}\left(A_{l}^{\prime}\right)_{I J}+\frac{v_{d}}{\sqrt{2}}\left(A_{l}\right)_{I J}, \\
& \left(\mathcal{M}_{L}^{2}\right)_{R R}=\frac{g_{1}^{2}\left(v_{u}^{2}-v_{d}^{2}\right)}{4} \delta_{I J}-g_{L}^{2}\left(\bar{v}_{L}^{2}-v_{L}^{2}\right) \delta_{I J}+m_{l^{l}}^{2} \delta_{I J}+\left(m_{\tilde{R}}^{2}\right)_{I J} .
\end{aligned}
$$

Then the mass matrix can be rotated to the mass eigenstates by the unitary matrix $Z_{\tilde{L}}$.

In the BLMSSM, the introduced superfields $\hat{N}^{c}$ lead to corrections for the couplings existing in MSSM. We deduce some corrected couplings, such as $W$-lepton-neutrino and $Z$-neutrino-neutrino couplings, which are shown as

$$
\begin{aligned}
& \mathcal{L}_{W l \nu}=-\frac{e}{\sqrt{2} s_{W}} W_{\mu}^{+} \sum_{I=1}^{3} \sum_{\alpha=1}^{6} Z_{N_{\nu}}^{I \alpha *} \bar{\chi}_{N_{\alpha}}^{0} \gamma^{\mu} P_{L} l^{I}, \\
& \mathcal{L}_{Z \nu \nu}=-\frac{e}{2 s_{W} c_{W}} Z_{\mu} \sum_{I=1}^{3} \sum_{\alpha, \beta=1}^{6} Z_{N_{\nu}}^{I \alpha *} Z_{N_{\nu}}^{I \beta} \bar{\chi}_{N_{\alpha}}^{0} \gamma^{\mu} P_{L} \chi_{N_{\beta}}^{0},
\end{aligned}
$$

where $P_{L}=\frac{1-\gamma_{5}}{2}$ and $P_{R}=\frac{1+\gamma_{5}}{2}$. We define $s_{W}=\sin \theta_{W}$, $c_{W}=\cos \theta_{W}$, and $\theta_{W}$ is the Weinberg angle.
The Z-sneutrino-sneutrino coupling is deduced as

$\mathcal{L}_{Z \tilde{\nu} \tilde{\nu}}=-\frac{e}{2 s_{W} c_{W}} Z_{\mu} \sum_{I=1}^{3} \sum_{i, j=1}^{6} Z_{\nu}^{I *} Z_{\nu}^{I j} \tilde{\nu}^{i *} i\left(\vec{\partial}^{\mu}-\overleftarrow{\partial}^{\mu}\right) \tilde{\nu}^{j}$

We also obtain the chargino-lepton-sneutrino coupling:

$$
\begin{aligned}
\mathcal{L}_{\chi^{ \pm} I \tilde{\nu}}= & -\sum_{I=1}^{3} \sum_{i=1}^{6} \sum_{j=1}^{2} \bar{\chi}_{j}^{-}\left(Y_{l}^{I} Z_{-}^{2 j *} Z_{\nu}^{I i *} P_{R}+\left[\frac{e}{s_{W}} Z_{+}^{1 j} Z_{\nu}^{I i *}\right.\right. \\
& \left.\left.+Y_{\nu}^{I i} Z_{+}^{2 j} Z_{\nu}^{(I+3) i *}\right] P_{L}\right) l^{I} \tilde{\nu}^{i *}+\text { H.c. }
\end{aligned}
$$

Considering the interactions of gauge and matter multiplets $i g \sqrt{2} T_{i j}^{a}\left(\lambda^{a} \psi_{j} A_{i}^{*}-\bar{\lambda}^{a} \bar{\psi}_{i} A_{j}\right)$, we deduce a new 




(a)

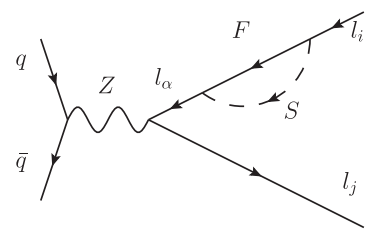

(e)

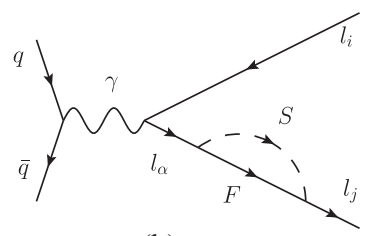

(b)

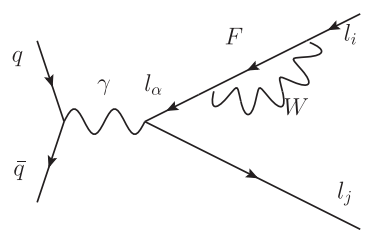

(c)

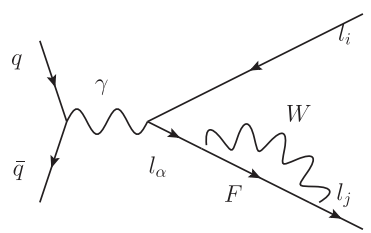

(d)

FIG. 1. The self-energy-type diagrams for processes $V \rightarrow l_{i}^{ \pm} l_{j}^{\mp}$, with $q$ representing $u, c, d, s, b$.

coupling for the lepton-slepton-lepton neutralino. The corresponding form for this coupling is written as

$\mathcal{L}_{l \chi_{L}^{0} \tilde{L}}=\sqrt{2} g_{L} \bar{\chi}_{L_{j}}^{0}\left(Z_{N_{L}}^{1 j} Z_{\tilde{L}}^{I i} P_{L}-Z_{N_{L}}^{1 *} Z_{\tilde{L}}^{(I+3) i} P_{R}\right) l^{I} \tilde{L}_{i}^{+}+$H.c.

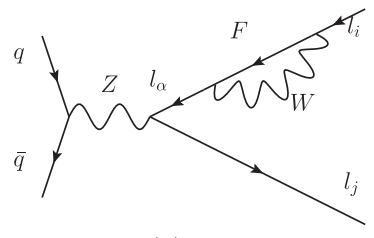

(g)

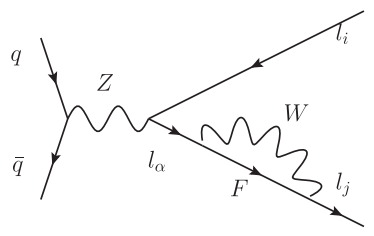

(h)

\section{THE AMPLITUDES FOR CLFV DECAYS OF VECTOR MESONS}

In the BLMSSM, the CLFV decays of vector mesons $V \rightarrow l_{i}^{ \pm} l_{j}^{\mp}$ with $V \in\left\{\phi, J / \Psi, \Upsilon, \rho^{0}, \omega\right\} \quad$ and $\quad l_{i}, \quad l_{j} \in$ $\{e, \mu, \tau\}$ are studied. We know that the meson consists of the quark and antiquark. The meson $\phi$ is made up of $s \bar{s}$; $J / \Psi$ is constituted of $c \bar{c} ; \Upsilon$ is composed of $b \bar{b} ; \rho^{0}$ is

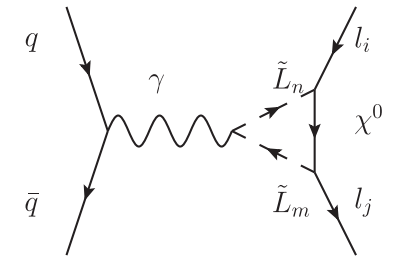

(a)

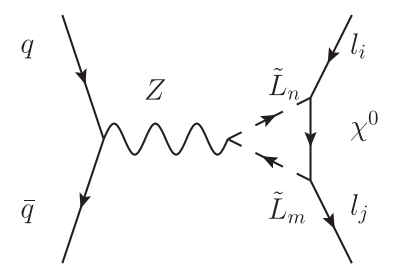

(e)

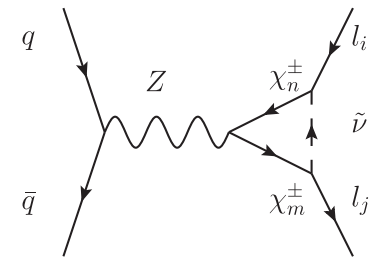

(i)

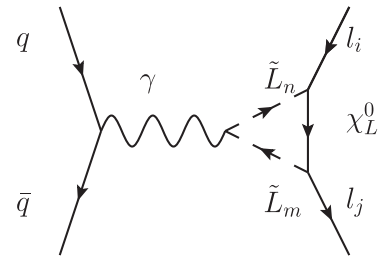

(b)

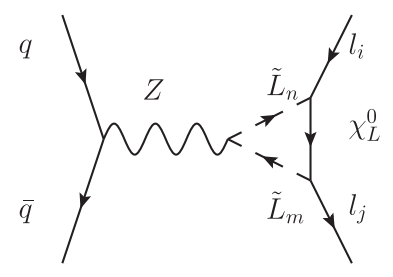

(f)

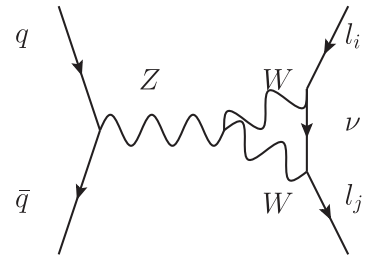

(j)

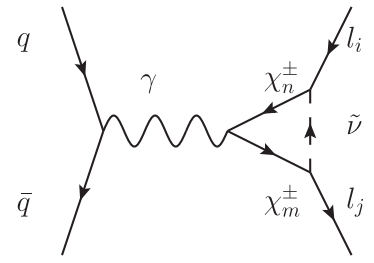

(c)

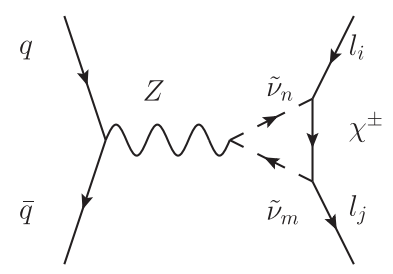

(g)

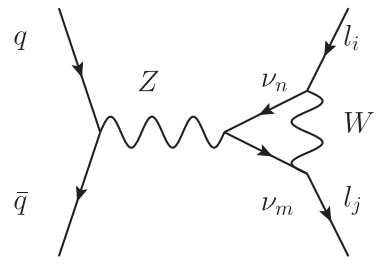

(k)

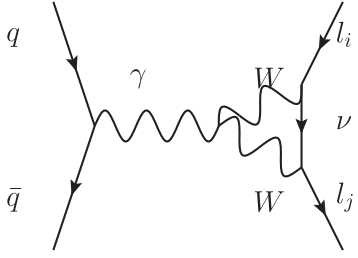

(d)

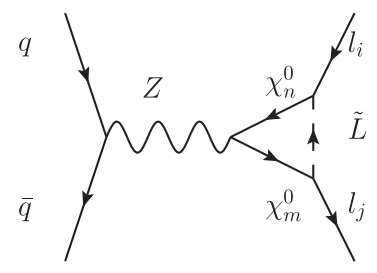

(h)

FIG. 2. The penguin-type diagrams for processes $V \rightarrow l_{i}^{ \pm} l_{j}^{\mp}$. 

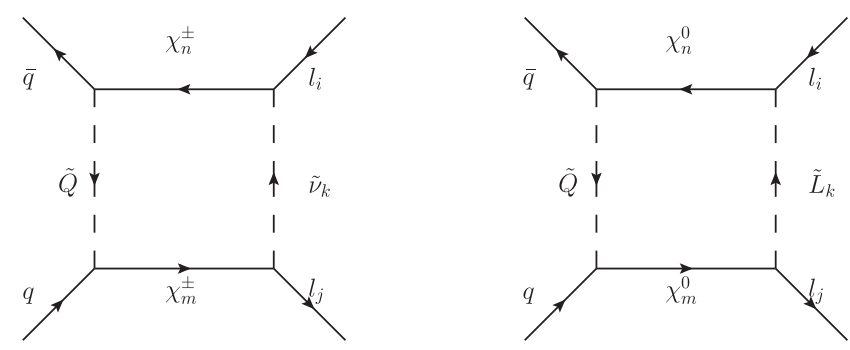

FIG. 3. The box type diagrams for processes $V \rightarrow l_{i}^{ \pm} l_{j}^{\mp}$.

comprised of $\frac{1}{\sqrt{2}}(u \bar{u}-d \bar{d})$, and $\omega$ consists of $\frac{1}{\sqrt{2}}(u \bar{u}+d \bar{d})$. We depict the relevant Feynman diagrams contributing to these processes in Figs. 1, 2, and 3.

In the quark picture, mesons are composed of a quark and an antiquark. QCD has the property of "quark confinement," so the traditional way to the perturbative calculation cannot work. At the quark-gluon level, the complicated calculation of loop integrations is governed by the nonperturbative QCD effects. However, a completely reliable way to these nonperturbative QCD effects is lacking at present. Therefore, as a powerful phenomenological model, a sum rule for the light-cone wave function [41-45] is adopted, which is widely used in the theoretical research of particle physics and nuclear physics.

To obtain the decay amplitude of processes involving a vector meson, one needs to calculate the matrix elements of gauge invariant nonlocal operators $[44,45]$,

$$
\langle 0|\bar{q}(y) \Gamma[x, y] q(x)| V\rangle
$$

where $\Gamma[x, y]$ is a generic Dirac matrix structure, and $x$ and $y$ represent the coordinates of quark and antiquark. The leading-twist distribution amplitude of the vector meson $V$ can be defined by the correlator $[44,45]$

$$
\begin{aligned}
& \left\langle 0\left|\bar{q}_{\alpha}(y) q_{\beta}(x)\right| V(p)\right\rangle \\
& =\frac{\delta_{i j}}{4 N_{c}} \int_{0}^{1} d u e^{-i(u p y+\bar{u} p x)}\left[f_{V} m_{V} \phi_{V} V_{\|}(u)\right. \\
& \left.\quad+\frac{i}{2} \sigma^{\mu^{\prime} \nu^{\prime}} f_{V}^{\top}\left(\varepsilon_{V \mu^{\prime}} p_{\nu^{\prime}}-\varepsilon_{V \nu^{\prime}} p_{\mu^{\prime}}\right) V_{\perp}(u)\right]_{\beta \alpha},
\end{aligned}
$$

where $m_{V}$ and $\varepsilon_{V}$ are, respectively, the mass and polarization vector of the vector meson, $f_{V}$ and $f_{V}^{\top}$ are the meson decay constants, and $V_{\|}(u)$ and $V_{\perp}(u)$ represent the leading-twist distribution functions corresponding to the longitudinally and transversely polarized mesons. The momentum $p$ satisfies $p^{2}=m_{V}^{2}$, which shows that the meson momentum is on-shell. The integration variable $u$ stands for the momentum fraction carried by the quark, and $\bar{u} \equiv 1-u$ corresponds to the momentum fraction of the antiquark. In our calculation, we make $V_{\|}=V_{\perp}=$ $V(u)=6 u(1-u)$, the reason is that the meson amplitudes are similar to their asymptotic form[46]. We take the number of colors $N_{c}=3$. The corresponding hadron matrix elements we used in our work are discussed in Appendix.

In the frame of center of mass, the amplitude of decay $V \rightarrow l_{i}^{ \pm} l_{j}^{\mp}$ can be shown at hadron level.

\section{A. Decays for self-energy type diagrams}

We can generally write the effective amplitudes of $\gamma$ lepton self-energy type diagrams corresponding to Figs. 1(a)-1(d):

$$
\begin{aligned}
\mathcal{A}_{\gamma-s}= & \bar{u}_{j}\left(p_{4}\right) \gamma_{\mu}\left(A_{L} P_{L}+A_{R} P_{R}\right) v_{i}\left(p_{3}\right) \\
& \times \frac{e}{p^{2}} \bar{v}_{V}\left(p_{2}\right) H_{V}^{\gamma q \bar{q}} \gamma^{\mu} u_{V}\left(p_{1}\right)
\end{aligned}
$$

where $p$ represents the momentum corresponding to meson $V, p_{1}$ and $p_{2}$ represent the quark and antiquark momentums, respectively, $p_{3}$ and $p_{4}$ represent the lepton and antilepton momentums, respectively. $\bar{u}_{j}$ and $v_{i}$ are the wave functions for the external leptons, with $i(j)$ representing the $i$ th- (jth-) generation charged lepton. As well as, $\bar{v}_{V}$ and $u_{V}$ are the wave functions for the external quarks, with $V$ representing mesons, here, $V \in\left\{\phi, J / \Psi, \Upsilon, \rho^{0}, \omega\right\} . H_{V}^{\gamma q \bar{q}}$ are the corresponding couplings for the different mesons. The Wilson coefficients $A_{L}$ and $A_{R}$ are shown as follows.

$A_{L, R}(S)$ stand for the contributions from Figs. 1(a) and 1(b), which are obtained with the sum of these diagrams' amplitudes.

$$
\begin{aligned}
A_{L}(S)= & \sum_{F=\chi^{0} / \chi_{L}^{0}, \chi^{ \pm}} \sum_{S=\tilde{L}, \tilde{L}} \frac{1}{x_{i}-x_{j}}\left\{-2 \sqrt{x_{F} x_{i}} H_{R}^{\bar{l}_{j} F S} H_{R}^{l_{i} \bar{F} S^{*}} I_{1}\left(x_{F}, x_{S}\right)+\left[( x _ { i } \sqrt { x _ { F } x _ { j } } H _ { L } ^ { \overline { l } _ { j } F S } H _ { L } ^ { l _ { i } \overline { F } S ^ { * } } - x _ { j } \sqrt { x _ { F } x _ { i } } H _ { R } ^ { \overline { l } _ { j } F S } H _ { R } ^ { l _ { i } \overline { F } S ^ { * } } ] \left[I_{2}\left(x_{F}, x_{S}\right)\right.\right.\right. \\
& \left.\left.-I_{3}\left(x_{F}, x_{S}\right)\right]+\left[\frac{1}{2}\left(x_{j}-x_{i}\right) H_{R}^{\bar{l}_{j} F S} H_{L}^{l_{i} \bar{F} S^{*}}+\sqrt{x_{i} x_{j}} H_{L}^{\bar{l}_{j} F S} H_{R}^{l_{i} \bar{F} S^{*}}\right] I_{5}\left(x_{F}, x_{S}\right)\right\}
\end{aligned}
$$

$A_{R}(S)=\left.A_{L}(S)\right|_{L \leftrightarrow R}$,

with $x=m^{2} / \Lambda^{2}, m$ is the mass of the corresponding particle and $\Lambda$ is the energy scale of the new physics.

The virtual $W$ diagrams Figs. 1(c) and 1(d) have contributions to the meson decay processes. The corresponding couplings $A_{L, R}(W)$ are shown as 


$$
A_{L}(W)=\sum_{F=\nu} \frac{x_{i}+x_{j}}{x_{i}-x_{j}} H_{L}^{\bar{l}_{j} F W} H_{L}^{l_{i} \bar{F} W^{*}} I_{5}\left(x_{F}, x_{W}\right), \quad A_{R}(W)=0
$$

In the same way, we deduced the effective amplitudes of the Z-lepton self-energy-type diagrams of Figs. 1(e)-1(h),

$$
\mathcal{A}_{Z-s}=\bar{u}_{j}\left(p_{4}\right) \gamma_{\mu}\left(B_{L} P_{L}+B_{R} P_{R}\right) v_{i}\left(p_{3}\right) \frac{1}{p^{2}-m_{Z}^{2}} \bar{v}_{V}\left(p_{2}\right) \gamma^{\mu}\left(H_{L}^{Z q \bar{q}} P_{L}+H_{R}^{Z q \bar{q}} P_{R}\right) u_{V}\left(p_{1}\right)
$$

The contributions from Figs. 1(e) and 1(f) are denoted by $B_{L, R}(S)$. We give out the concrete forms as follows,

$$
\begin{aligned}
B_{L}(S)= & \sum_{F=\chi^{0} / \chi_{L}^{0}, \chi^{ \pm}} \sum_{S=\tilde{L}, \tilde{\nu}} \frac{1}{x_{i}-x_{j}}\left\{-2 \sqrt{x_{F} x_{i}} H_{L}^{Z \bar{l}_{i} l_{j}} H_{R}^{\bar{l}_{j} F S} H_{R}^{l_{i} \bar{F} S^{*}} I_{1}\left(x_{F}, x_{S}\right)\right. \\
+ & {\left[\left(x_{i} \sqrt{x_{F} x_{j}} H_{L}^{Z \bar{l}_{i} l_{j}} H_{L}^{\bar{l}_{j} F S} H_{L}^{l_{i} \bar{F} S^{*}}-x_{j} \sqrt{x_{F} x_{i}} H_{L}^{Z \bar{l}_{i} l_{j}} H_{R}^{\bar{l}_{j} F S} H_{R}^{l_{i} \bar{F} S^{*}}\right]\left[I_{2}\left(x_{F}, x_{S}\right)-I_{3}\left(x_{F}, x_{S}\right)\right]\right.} \\
+ & {\left.\left[\frac{1}{2}\left(x_{j}-x_{i}\right) H_{L}^{Z \bar{l}_{i} l_{j}} H_{R}^{\bar{l}_{j} F S} H_{L}^{l_{i} \bar{F} S^{*}}+\sqrt{x_{i} x_{j}} H_{L}^{Z \bar{l}_{i} l_{j}} H_{L}^{\bar{l}_{j} F S} H_{R}^{l_{i} \bar{F} S^{*}}\right] I_{5}\left(x_{F}, x_{S}\right)\right\} } \\
B_{R}(S)= & \left.B_{L}(S)\right|_{L \leftrightarrow R} .
\end{aligned}
$$

The corresponding coefficients for Figs. $1(\mathrm{~g})$ and $1(\mathrm{~h})$ are described by $B_{L, R}(W)$,

$$
B_{L}(W)=\sum_{F=\nu} \frac{x_{i}+x_{j}}{x_{i}-x_{j}} H_{L}^{Z \bar{l}_{i} l_{j}} H_{L}^{\bar{l}_{j} F W} H_{L}^{l_{i} \bar{F} W^{*}} I_{5}\left(x_{F}, x_{W}\right), \quad B_{R}(W)=0 .
$$

\section{B. Decays for penguin-type diagrams}

In Fig. 2, we give out the corresponding penguin-type diagrams for decays $V \rightarrow l_{i}^{ \pm} l_{j}^{\mp}$. When the external leptons are all on-shell, we obtain the effective amplitudes of $\gamma$ penguin-type diagrams,

$$
\mathcal{A}_{\gamma-p}=\bar{u}_{j}\left(p_{4}\right)\left[\gamma_{\mu}\left(C_{1}^{L} P_{L}+C_{1}^{R} P_{R}\right)+i \sigma_{\mu \nu} p^{\nu}\left(C_{2}^{L} P_{L}+C_{2}^{R} P_{R}\right)\right] v_{i}\left(p_{3}\right) \times \frac{e}{p^{2}} \bar{v}_{V}\left(p_{2}\right) H_{V}^{\gamma q \bar{q}} \gamma^{\mu} u_{V}\left(p_{1}\right)
$$

$C_{1}^{L, R}(n), C_{2}^{L, R}(n)$ represent the contributions from the virtual neutral fermion diagrams Figs. 2(a) and 2(b):

$$
\begin{aligned}
& C_{1}^{L}(n)=\sum_{F=\chi^{0} / \chi_{L}^{0}} \sum_{S=\tilde{L}} H_{R}^{S F \bar{l}_{j}} H_{L}^{S^{*} l_{i} \bar{F}}\left[\frac{1}{2} I_{5}\left(x_{F}, x_{S}\right)+\frac{p^{2}}{6 \Lambda^{2}} I_{4}\left(x_{F}, x_{S}\right)\right], \\
& C_{2}^{L}(n)=\sum_{F=\chi^{0} / \chi_{L}^{0}} \sum_{S=\tilde{L}} \frac{m_{F}}{\Lambda^{2}} H_{L}^{S F \bar{l}_{j}} H_{L}^{S^{*} l_{i} \bar{F}}\left[I_{2}\left(x_{F}, x_{S}\right)-I_{3}\left(x_{F}, x_{S}\right)\right], \\
& C_{\alpha}^{R}(n)=\left.C_{\alpha}^{L}(n)\right|_{L \leftrightarrow R}, \quad \alpha=1,2 .
\end{aligned}
$$

The couplings coefficients $C_{1}^{L, R}(c), C_{1}^{L, R}(c)$ for Fig. 2(c) are written as

$$
\begin{aligned}
& C_{1}^{L}(c)=\sum_{F=\chi^{ \pm}} \sum_{S=\tilde{\nu}} H_{R}^{S F \bar{l}_{j}} H_{L}^{S^{*} l_{i} \bar{F}}\left[\frac{1}{2} I_{5}\left(x_{S}, x_{F}\right)+x_{F} I_{3}\left(x_{S}, x_{F}\right)+\frac{p^{2}}{6 \Lambda^{2}}\left(3 I_{2}\left(x_{S}, x_{F}\right)-I_{4}\left(x_{S}, x_{F}\right)\right)\right], \\
& C_{2}^{L}(c)=-\sum_{F=\chi^{ \pm}} \sum_{S=\tilde{\nu}} \frac{m_{F}}{\Lambda^{2}} H_{L}^{S F \bar{l}_{j}} H_{L}^{S^{*} l_{i} \bar{F}} I_{2}\left(x_{S}, x_{F}\right), \quad C_{\alpha}^{R}(c)=\left.C_{\alpha}^{L}(c)\right|_{L \leftrightarrow R}, \alpha=1,2 .
\end{aligned}
$$


We also acquire the contributions $C_{1}^{L, R}(W), C_{1}^{L, R}(W)$ from Fig. 2(d), which are in the following forms:

$$
C_{1}^{L}(W)=\sum_{F=\nu} H_{L}^{W F \bar{l}_{j}} H_{L}^{W^{*} l_{i} \bar{F}}\left[I_{5}\left(x_{F}, x_{W}\right)+\frac{p^{2}}{\Lambda^{2}}\left(-2 I_{2}\left(x_{F}, x_{W}\right)+\frac{1}{3} I_{4}\left(x_{F}, x_{W}\right)\right)\right], \quad C_{2}^{L}(W)=C_{\alpha}^{R}(W)=0, \quad \alpha=1,2 .
$$

Using the same method as the $\gamma$ penguin-type diagrams, the effective Lagrangian for the $Z$ penguin-type diagrams is deduced as follows. Here, we just give out the dominate contributions of the effective operators:

$$
\mathcal{A}_{z-p}=\bar{u}_{j}\left(p_{4}\right) \gamma_{\mu}\left(D_{L} P_{L}+D_{R} P_{R}\right) v_{i}\left(p_{3}\right) \frac{1}{p^{2}-m_{Z}^{2}} \bar{v}\left(p_{2}\right) \gamma^{\mu}\left(H_{L}^{z q \bar{q}} P_{L}+H_{R}^{z q \bar{q}} P_{R}\right) u\left(p_{1}\right)
$$

The concrete contributions for $D_{L, R}(S)$ are encoded as follows, which correspond to Figs. 2(e)-2(i):

$$
\begin{aligned}
D_{L}(S)= & -\frac{1}{2} \sum_{F=\chi^{0} \cdot \chi^{ \pm}} \sum_{S=\tilde{L}, \tilde{\nu}}\left[-2 \sqrt{x_{F_{1}} x_{F_{2}}} H_{L}^{S F_{2} \bar{l}_{j}} H_{R}^{Z F_{1} \bar{F}_{2}} H_{L}^{S^{*} l_{i} \bar{F}_{1}} G_{1}\left(x_{S}, x_{F_{1}}, x_{F_{2}}\right)+H_{R}^{S F_{2} \bar{l}_{j}} H_{R}^{Z F_{1} \bar{F}_{2}} H_{L}^{S^{*} l_{i} \bar{F}_{1}} G_{2}\left(x_{S}, x_{F_{1}}, x_{F_{2}}\right)\right. \\
& \left.-H_{R}^{S_{2} F \bar{l}_{j}} H^{Z S_{1} S_{2}^{*}} H_{L}^{S_{1}^{*} l_{i} \bar{F}} G_{2}\left(x_{F}, x_{S_{1}}, x_{S_{2}}\right)\right]+\frac{1}{2} \sum_{F=\chi_{L}^{0}} \sum_{S=\tilde{L}}\left[H_{R}^{S_{2} F \bar{l}_{j}} H^{Z S_{1} S_{2}^{*}} H_{L}^{S_{1}^{*} l_{l} \bar{F}} G_{2}\left(x_{F}, x_{S_{1}}, x_{S_{2}}\right)\right], \\
D_{R}(S)= & \left.D_{L}(S)\right|_{L \leftrightarrow R} .
\end{aligned}
$$

We also deduce the effective couplings $D_{L, R}(W)$ in detail, which are written as

$$
\begin{aligned}
D_{L}(W)= & \sum_{F=\nu} H_{L}^{W F \bar{l}_{j}} H^{Z W W^{*}} H_{L}^{W^{*} l_{i} \bar{F}}\left[I_{5}\left(x_{F}, x_{W}\right)+\frac{p^{2}}{\Lambda^{2}}\left(-2 I_{2}\left(x_{F}, x_{W}\right)+\frac{1}{3} I_{4}\left(x_{F}, x_{W}\right)\right)\right] \\
& -H_{L}^{W F_{2} \bar{l}_{j}} H^{Z F_{2} \bar{F}_{2}} H_{L}^{W^{*} l_{i} \bar{F}_{1}} G_{2}\left(x_{W}, x_{F_{1}}, x_{F_{2}}\right), \quad D_{R}(W)=0 .
\end{aligned}
$$

Then, we discuss the contributions of the box-type diagrams in Fig. 3. To simplify the corresponding amplitudes, we need to swap the position of the wave functions $u\left(p_{1}\right)$ and $v_{i}\left(p_{3}\right)$. The method is named as Fierz rearrangement, and the corresponding transformation rules and characters can be learned from Refs. [47-49]. After that, the reduced results of the amplitudes can be written as

$$
\begin{aligned}
\mathcal{A}_{\mathrm{box}}= & \left\{N_{1}^{L} \bar{u}_{j}\left(p_{4}\right) \gamma_{\mu} P_{L} v_{i}\left(p_{3}\right) \bar{v}\left(p_{2}\right) \gamma^{\mu} P_{L} u\left(p_{1}\right)+(L \leftrightarrow R)\right\}+\left\{N_{2}^{L}\left[\bar{u}_{j}\left(p_{4}\right) \sigma_{\mu \nu} v_{i}\left(p_{3}\right) \bar{v}\left(p_{2}\right) \sigma^{\mu \nu} P_{L} u\left(p_{1}\right)+(L \leftrightarrow R)\right\}\right. \\
& +\left\{N_{3}^{L}\left[\bar{u}_{j}\left(p_{4}\right) \gamma_{\mu} P_{R} v_{i}\left(p_{3}\right) \bar{v}\left(p_{2}\right) \gamma^{\mu} P_{L} u\left(p_{1}\right)+(L \leftrightarrow R)\right\},\right.
\end{aligned}
$$

where

$$
\begin{aligned}
& N_{1}^{L}=\frac{1}{4} \sum_{F_{1}, F_{2}=\chi^{0} \cdot \chi^{ \pm}} \sum_{S_{1}=\tilde{Q}} \sum_{S_{2}=\tilde{L}, \tilde{\nu}} H_{L}^{q S_{1} \bar{F}_{2}} H_{R}^{\bar{q} S_{1}^{*} F_{1}} H_{L}^{l_{i} S_{2} \bar{F}_{1}} H_{R}^{\bar{l}_{j} S_{2}^{*} F_{2}} J_{1}\left(x_{F_{1}}, x_{F_{2}}, x_{S_{1}}, x_{S_{2}}\right), \\
& N_{2}^{L}=-\frac{\sqrt{x_{F_{1}} x_{F_{2}}}}{8} \sum_{F_{1}, F_{2}=\chi^{0}, \chi^{ \pm}} \sum_{S_{1}=\tilde{Q}} \sum_{S_{2}=\tilde{L}, \tilde{\nu}} H_{L}^{q S_{1} \bar{F}_{2}} H_{L}^{\bar{q} S_{1}^{*} F_{1}} H_{L}^{l_{i} S_{2} \bar{F}_{1}} H_{L}^{\bar{l}_{i} S_{2}^{*} F_{2}} J_{2}\left(x_{F_{1}}, x_{F_{2}}, x_{S_{1}}, x_{S_{2}}\right), \\
& N_{3}^{L}=-\frac{\sqrt{x_{F_{1}} x_{F_{2}}}}{2} \sum_{F_{1}, F_{2}=\chi^{0}, \chi^{ \pm}} \sum_{S_{1}=\tilde{Q}} \sum_{S_{2}=\tilde{L}, \tilde{\nu}} H_{L}^{q S_{1} \bar{F}_{2}} H_{R}^{\bar{q} S_{1}^{*} F_{1}} H_{R}^{l_{i} S_{2} \bar{F}_{1}} H_{L}^{\bar{l}_{j} S_{2}^{*} F_{2}} J_{2}\left(x_{F_{1}}, x_{F_{2}}, x_{S_{1}}, x_{S_{2}}\right) .
\end{aligned}
$$

In Eqs. (18)-(32), $H_{V}^{\gamma q \bar{q}}, H_{L, R}^{\bar{l}_{j} F S}, \ldots$ represent the corresponding couplings in the Lagrangian. The one-loop functions $I_{i}\left(x_{1}, x_{2}\right), i=1,2, \ldots, 5, G_{i}\left(x_{1}, x_{2}, x_{3}\right), i=1,2$, and $J_{i}\left(x_{1}, x_{2}, x_{3}, x_{4}\right), i=1,2$ are shown in the Appendix.

The branching ratios for processes $V \rightarrow l_{i}^{ \pm} l_{j}^{\mp}$ can be deduced as

$$
\operatorname{Br}\left(V \rightarrow l_{i}^{ \pm} l_{j}^{\mp}\right)=\frac{\sqrt{\left[m_{V}^{2}-\left(m_{l_{i}}+m_{l_{j}}\right)^{2}\right]\left[m_{V}^{2}-\left(m_{l_{i}}-m_{l_{j}}\right)^{2}\right]}}{16 \pi m_{V}^{3} \Gamma_{V}} \sum_{\xi} \mathcal{A}_{V_{\xi}} \mathcal{A}_{V_{\xi}}^{*},
$$


where $\Gamma_{V}$ represents the total decay width of meson $V$ (with $V \in\left\{\phi, J / \Psi, \Upsilon, \rho^{0}, \omega\right\}$ and $\left.l_{i}, l_{j} \in\{e, \mu, \tau\}\right)$. We chose $\Gamma_{\phi} \simeq 4.2 \times 10^{-3} \mathrm{GeV}, \quad \Gamma_{J / \Psi} \simeq 0.093 \times 10^{-3} \mathrm{GeV}, \quad \Gamma_{\Upsilon} \simeq$ $0.054 \times 10^{-3} \mathrm{GeV}, \Gamma_{\rho^{0}} \simeq 0.149 \mathrm{GeV}, \Gamma_{\omega} \simeq 8.49 \times 10^{-3} \mathrm{GeV}$ [29]. $A_{V_{\xi}}$ are the amplitudes corresponding to Figs. 1, 2, and 3. Summation formula $\sum_{\lambda= \pm 1,0} \varepsilon_{\lambda}^{\mu}(p) \varepsilon_{\lambda}^{* \nu}(p)=-g^{\mu \nu}+$ $\frac{p^{\mu} p^{\nu}}{m_{V}^{2}}$ can be used to simplify $\sum_{\xi} A_{V_{\xi}} A_{V_{\xi}}^{*}$.

\section{NUMERICAL RESULTS}

In the numerical analysis, we consider the experimental constraints from the light neutral Higgs mass $m_{h^{0}} \simeq$ $125 \mathrm{GeV}$ [50-52] and the neutrino experiment data $[1,4,5,53,54]$,

$$
\begin{aligned}
\sin ^{2} \theta_{13} & =(2.19 \pm 0.12) \times 10^{-2}, \\
\sin ^{2} \theta_{12} & =0.304 \pm 0.014, \\
\sin ^{2} \theta_{23} & =0.51 \pm 0.05, \\
\Delta m_{\odot}^{2} & =(7.53 \pm 0.18) \times 10^{-5} \mathrm{eV}^{2}, \\
\left|\Delta m_{A}^{2}\right| & =(2.44 \pm 0.06) \times 10^{-3} \mathrm{eV}^{2} .
\end{aligned}
$$

In our previous works, $\operatorname{Br}(\mu \rightarrow e \gamma)<5.7 \times 10^{-13}, \operatorname{Br}(\mu \rightarrow$ $3 e)<1.0 \times 10^{-12}$, and $\operatorname{Br}(Z \rightarrow e \mu)<7.5 \times 10^{-7} \quad[24,29,55]$ are strict constraints for our parameter space. In this work, we take the same parameter space satisfying the constraints of processes $\mu \rightarrow e \gamma, \mu \rightarrow 3 e$, and $Z \rightarrow e \mu$.

In this work, the meson masses are adopted as $m_{\phi}=1.019, m_{J / \Psi}=3.096, m_{\Upsilon}=9.460, m_{\rho^{0}}=0.775$, and $m_{\omega}=0.782 \mathrm{GeV}$. The decay constants for the corresponding mesons are shown as $f_{\phi}=0.231, f_{J / \Psi}=0.405$, $f_{\Upsilon}=0.715, f_{\rho^{0}}=0.209$, and $f_{\omega}=0.195 \mathrm{GeV}$. Furthermore, some other parameters we used are shown as follows $[29,56]:$

$$
\begin{aligned}
m_{e} & =0.51 \times 10^{-3} \mathrm{GeV}, \quad m_{\mu}=0.105 \mathrm{GeV}, \\
m_{\tau} & =1.777 \mathrm{GeV}, \quad L_{4}=\frac{3}{2}, \quad m_{u}=2.2 \times 10^{-3} \mathrm{GeV}, \\
m_{c} & =1.27 \mathrm{GeV}, \quad m_{t}=173.2 \mathrm{GeV}, \quad B_{4}=\frac{3}{2}, \\
m_{d} & =4.7 \times 10^{-3} \mathrm{GeV}, \quad m_{s}=0.096 \mathrm{GeV}, \\
m_{b} & =4.18 \mathrm{GeV}, \quad \lambda_{N^{c}}=1, \quad m_{Z}=91.1876 \mathrm{GeV}, \\
m_{W} & =80.385 \mathrm{GeV}, \quad \alpha\left(m_{Z}\right)=1 / 128, \\
s_{W}^{2}\left(m_{Z}\right) & =0.23, \quad\left(Y_{\nu}\right)_{11}=1.3031 * 10^{-6}, \\
\left(Y_{\nu}\right)_{12} & =9.0884 * 10^{-8}, \quad\left(Y_{\nu}\right)_{13}=6.9408 * 10^{-8}, \\
\left(Y_{\nu}\right)_{22} & =1.6002 * 10^{-6}, \quad\left(Y_{\nu}\right)_{23}=3.4872 * 10^{-7}, \\
\left(Y_{\nu}\right)_{33} & =1.7208 * 10^{-6} .
\end{aligned}
$$

We assume $\mu=0.7 \mathrm{TeV}, \mu_{L}=0.5 \mathrm{TeV}, \Lambda=M_{L}=$ $\left(A_{u}\right)_{i i}=\left(A_{d}\right)_{i i}=\left(A_{d}^{\prime}\right)_{i i}=\left(m_{\tilde{N}^{c}}\right)_{i i}=1 \mathrm{TeV}, \quad\left(A_{l}\right)_{i i}=$ $-2 \mathrm{TeV},\left(A_{N^{c}}\right)_{i i}=\left(A_{N}\right)_{i i}=0.5 \mathrm{TeV},\left(A_{u}^{\prime}\right)_{i i}=0.8 \mathrm{TeV}$,
$\left(A_{l}^{\prime}\right)_{i i}=A_{L}^{\prime}, \quad$ and $\quad\left(m_{\tilde{Q}}\right)_{i i}=\left(m_{\tilde{U}}\right)_{i i}=\left(m_{\tilde{D}}\right)_{i i}=2 \mathrm{TeV}$, where $i=1,2,3 \cdot \tan \beta_{L}=\bar{v}_{L} / v_{L}$ and $V_{L_{t}}=\sqrt{\bar{v}_{L}^{2}+v_{L}^{2}}$. The diagonal entries of matrices $m_{\tilde{L}}^{2}$ and $m_{\tilde{R}}^{2}$ are supposed as $\left(m_{\tilde{L}}^{2}\right)_{i i}=\left(m_{\tilde{R}}^{2}\right)_{i i}=S_{m}^{2}$ and nondiagonal terms $\left(m_{\tilde{L}}^{2}\right)_{i j}=$ $\left(m_{\tilde{R}}^{2}\right)_{i j}=M_{L_{f}}^{2}$, with $i \neq j$ and $i, j=1,2,3$. $m_{1}$ represents the gaugino mass in $U(1)$, and $m_{2}$ represents the gaugino mass in $S U(2)$. We take $m_{1}=0.5 \mathrm{TeV}$. Generally, if we do not specify, the nondiagonal elements of the parameters are defined as zero.

\section{A. $V \rightarrow \boldsymbol{e}^{+} \boldsymbol{\mu}^{-}$}

At first, we discuss the CLFV decays of vector mesons $V \rightarrow e^{+} \mu^{-}$with $V \in\left\{\phi, J / \Psi, \Upsilon, \rho^{0}, \omega\right\}$. The branching ratios for processes $\phi(J / \Psi) \rightarrow e^{+} \mu^{-}$are strict. The corresponding experimental limits are $\operatorname{Br}\left(\phi \rightarrow e^{+} \mu^{-}\right) \leq$ $2.0 \times 10^{-6}$ at $90 \%$ confidence level and $\operatorname{Br}\left(J / \Psi \rightarrow e^{+} \mu^{-}\right) \leq$ $1.6 \times 10^{-7}$ at $95 \%$ confidence level.

As the diagonal elements of the mass matrices $m_{\tilde{L}}^{2}$ and $m_{\tilde{R}}^{2}, S_{m}$ present in the mass matrices of slepton and sneutrino. And the CLFV processes can be influenced by slepton-neutralino, slepton-lepton neutralino, and chargino-sneutrino contributions. At this subsection, the parameters are supposed to be $\tan \beta=6, m_{2}=0.5 \mathrm{TeV}$, $g_{L}=0.1, \tan \beta_{L}=2, V_{L_{t}}=3 \mathrm{TeV}, A_{L}^{\prime}=0.3 \mathrm{TeV}$, and $M_{L_{f}}^{2}=2 * 10^{5} \mathrm{GeV}^{2}$. In Fig. 4 , we plot the branching ratios of decays $V \rightarrow e^{+} \mu^{-}$varying with $S_{m}$. Here, a different line corresponds to a different decay process. We find that the results for processes $J / \Psi \rightarrow e^{+} \mu^{-}$and $\Upsilon \rightarrow e^{+} \mu^{-}$are around $10^{-8} \sim 10^{-11}$, the results of $\phi \rightarrow$ $e^{+} \mu^{-}$are around $10^{-10} \sim 10^{-13}$, and the results of $\rho^{0} \rightarrow$ $e^{+} \mu^{-}$and $\omega \rightarrow e^{+} \mu^{-}$are around $10^{-11} \sim 10^{-14}$. These five lines all decrease quickly with the increasing $S_{m}$. Therefore, $S_{m}$ are very sensitive parameters to the numerical results.

The parameter $m_{2}$ not only presents in the mass matrix of the neutralino, but also in the mass matrix of the chargino. Therefore, $m_{2}$ affects the numerical results through the neutralino-slepton and chargino-sneutrino contributions.

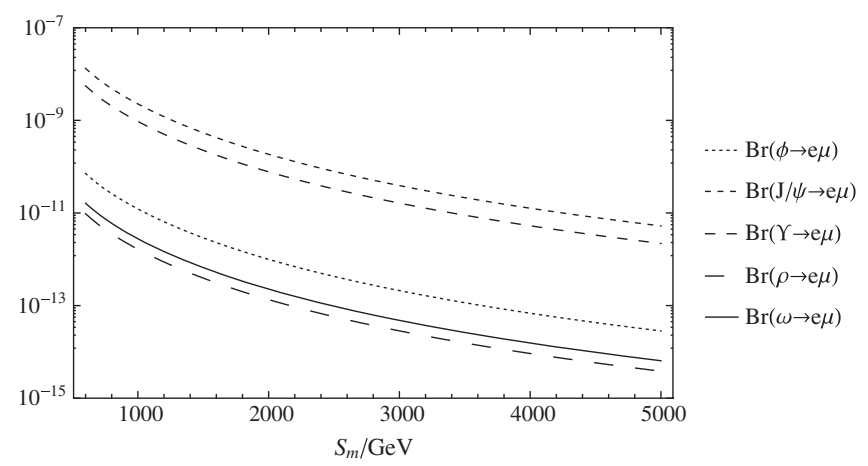

FIG. 4. The contributions to $V \rightarrow e^{+} \mu^{-}\left(V \in\left\{\phi, J / \Psi, \Upsilon, \rho^{0}, \omega\right\}\right)$ varying with $S_{m}$ are, respectively, plotted by the five lines. 


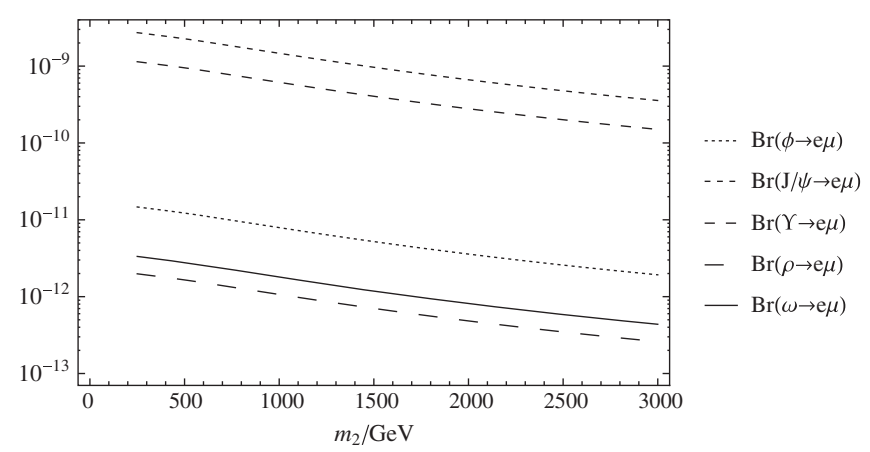

FIG. 5. The decays $V \rightarrow e^{+} \mu^{-}\left(V \in\left\{\phi, J / \Psi, \Upsilon, \rho^{0}, \omega\right\}\right)$ versus parameter $m_{2}$ are plotted by the five lines, respectively.

The branching ratios of $V \rightarrow e^{+} \mu^{-}$varying with parameter $m_{2}$ are shown in Fig. 5. Choosing $\tan \beta=6, g_{L}=0.1$, $\tan \beta_{L}=2, V_{L_{t}}=3 \mathrm{TeV}, A_{L}^{\prime}=0.3 \mathrm{TeV}, M_{L_{f}}^{2}=2 * 10^{5} \mathrm{GeV}^{2}$, and $S_{m}=1 \mathrm{TeV}$, with the enlarging $m_{2}$, the branching ratios for each process all decrease. However, the results do not change very remarkably. Although $m_{2}$ is a sensitive parameter, the influence from $m_{2}$ is smaller than that from $S_{m}$.

\section{1. $J / \Psi(\Upsilon) \rightarrow e^{+} \mu^{-}$}

Here, we study the decay processes $J / \Psi \rightarrow e^{+} \mu^{-}$and $\Upsilon \rightarrow e^{+} \mu^{-}$. The many mass matrix, such as sleptons, sneutrinos, neutrino, include the parameter $\tan \beta$, and the numerical results can be influenced by this parameter. Supposing $g_{L}=0.1, \quad \tan \beta_{L}=2, \quad V_{L_{t}}=3 \mathrm{TeV}$, $A_{L}^{\prime}=0.3 \mathrm{TeV}, M_{L_{f}}^{2}=2 * 10^{5} \mathrm{GeV}^{2}, S_{m}=1 \mathrm{TeV}$, and $m_{2}=0.5 \mathrm{TeV}$, we give out the values of $\operatorname{Br}(J / \Psi(\Upsilon) \rightarrow$ $\left.e^{+} \mu^{-}\right)$versus $\tan \beta$ in Fig. 6 . When the $\tan \beta$ changes from 0 to 10 , the numerical results decrease quickly. When $\tan \beta>10$, the numerical results almost do not change. Therefore, in the range of $0 \sim 10, \tan \beta$ have sensitive effects for the CLFV decays.

$M_{L_{f}}$ is of interest. As the nondiagonal elements of $m_{\tilde{L}}^{2}$ and $m_{\tilde{R}}^{2}, M_{L_{f}}$ lead to strong mixing for slepton (sneutrino) of different generations. So we study the processes with parameter $M_{L_{f}}$. With $\tan \beta=6, m_{2}=0.5 \mathrm{TeV}, g_{L}=0.1$,

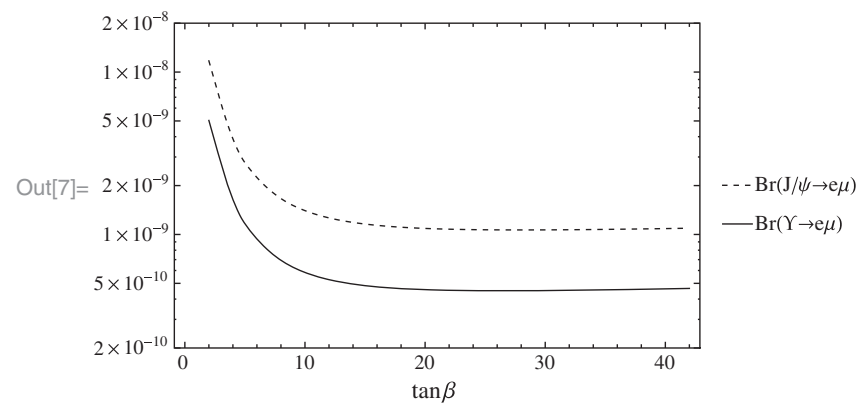

FIG. 6. The branching ratios of decays $J / \Psi(\Upsilon) \rightarrow e^{+} \mu^{-}$ change with the parameter $\tan \beta$.

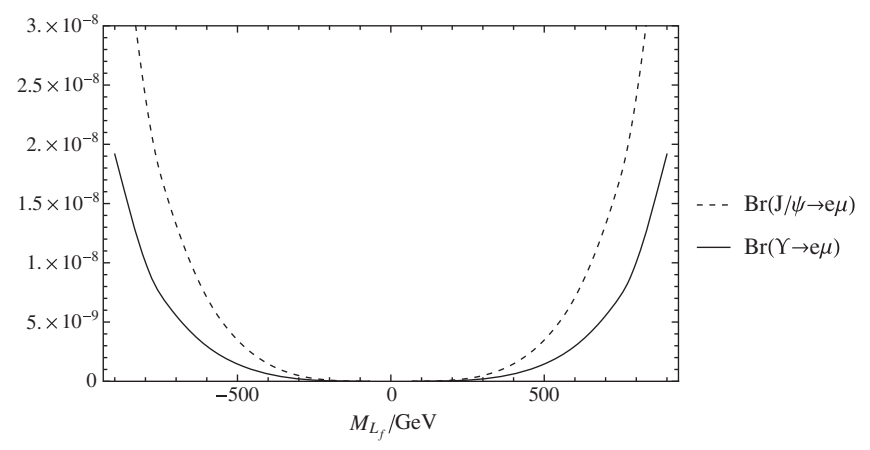

FIG. 7. The branching ratios for processes $J / \Psi(\Upsilon) \rightarrow e^{+} \mu^{-}$ change with the parameter $M_{L_{f}}$.

$\tan \beta_{L}=2, \quad V_{L_{t}}=3 \mathrm{TeV}, \quad A_{L}^{\prime}=0.3 \mathrm{TeV}, \quad$ and $\quad S_{m}=$ $1 \mathrm{TeV}$, the numerical results are plotted corresponding to parameter $M_{L_{f}}$ in Fig. 7 . When $M_{L_{f}}$ is zero, the branching ratios are almost zero; with enlarging $\left|M_{L_{f}}\right|$, the results for both processes increase quickly, which are around $0 \sim 10^{-8}$, and the figures are very symmetrical for both processes. Obviously, $M_{L_{f}}$ is a much more sensitive parameter, and the effects from $M_{L_{f}}$ are very strong for these CLFV decays.

$$
\text { 2. } \phi\left(\rho^{0}, \omega\right) \rightarrow e^{+} \mu^{-}
$$

As new parameters in the BLMSSM, $g_{L}$ and $\tan \beta_{L}$ have relations with the mass matrices of slepton, sneutrino, and lepton neutralino. It is worth considering the contributions from $g_{L}$ and $\tan \beta_{L}$. Based on the supposition $\tan \beta=6$, $m_{2}=0.5 \mathrm{TeV}, \tan \beta_{L}=2, V_{L_{t}}=3 \mathrm{TeV}, A_{L}^{\prime}=0.3 \mathrm{TeV}$, $S_{m}=1 \mathrm{TeV}$, and $M_{L_{f}}^{2}=2 * 10^{5} \mathrm{GeV}^{2}$, the branching ratios for $\phi\left(\rho^{0}, \omega\right) \rightarrow e^{+} \mu^{-}$are discussed with parameter $g_{L}$ in Fig. 8. The values for each process decrease slightly with the enlarging $g_{L}$, so the effects from $g_{L}$ on the numerical results are small.

The slepton mass squared matrix includes the parameter $A_{L}^{\prime}$, which is the nondiagonal element of this matrix.

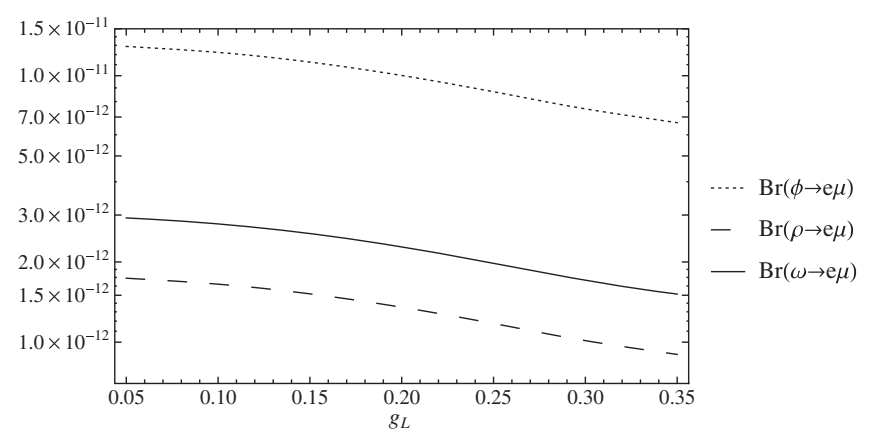

FIG. 8. The contributions to $\operatorname{Br}\left(\phi\left(\rho^{0}, \omega\right) \rightarrow e^{+} \mu^{-}\right)$varying with $g_{L}$ are plotted by the dotted line, dashed line, and solid line, respectively. 


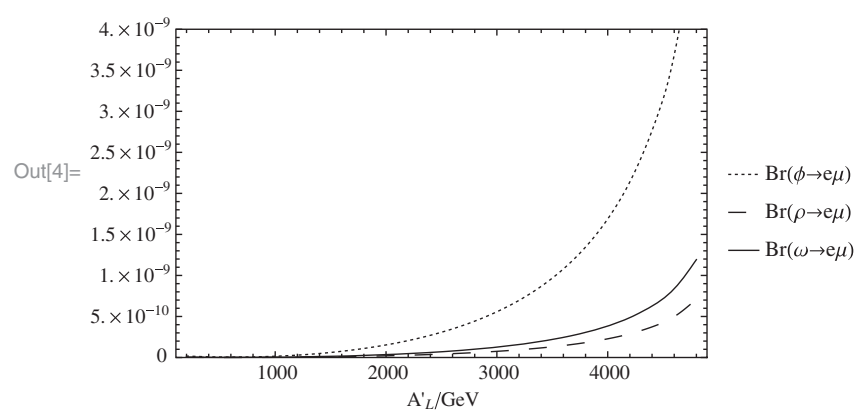

FIG. 9. The numerical results for $\operatorname{Br}\left(\phi\left(\rho^{0}, \omega\right) \rightarrow e^{+} \mu^{-}\right)$versus $A_{L}^{\prime}$ are plotted.

So the results can be affected by $A_{L}^{\prime}$ through slepton mass and mixing. Here, $\tan \beta=6, \quad m_{2}=0.5 \mathrm{TeV}$, $g_{L}=0.1, \quad \tan \beta_{L}=2, V_{L_{t}}=3 \mathrm{TeV}, \quad S_{m}=1 \mathrm{TeV}$, and $M_{L_{f}}^{2}=2 * 10^{5} \mathrm{GeV}^{2}$, the branching ratios for $\phi\left(\rho^{0}, \omega\right) \rightarrow$ $e^{+} \mu^{-}$versus parameter $A_{L}^{\prime}$ are plotted in Fig. 9. With $A_{L}^{\prime}$ changing from 0 to $3 \mathrm{TeV}$, both processes slightly increase. However, the numerical results change obviously when $A_{L}^{\prime}>3 \mathrm{TeV}$, especially the process $\phi \rightarrow e^{+} \mu^{-}$. In a reasonable range of parameter $A_{L}^{\prime}$, the branching ratios will be close to the experimental expectations with the enlarging $A_{L}^{\prime}$.

\section{B. $V \rightarrow e^{+} \tau^{-}\left(\mu^{+} \tau^{-}\right)$}

We study the decays $V \rightarrow e^{+} \tau^{-}\left(\mu^{+} \tau^{-}\right)$as follows, where $V \in\{J / \Psi, \Upsilon\}$. The experimental limits for decays $J / \Psi \rightarrow e^{+} \tau^{-}\left(\mu^{+} \tau^{-}\right)$are $\operatorname{Br}\left(J / \Psi \rightarrow e^{+} \tau^{-}\right) \leq 8.3 \times 10^{-6}$ and $\operatorname{Br}\left(J / \Psi \rightarrow \mu^{+} \tau^{-}\right) \leq 2.0 \times 10^{-6}$, which are both at $90 \%$ confidence level. The experimental limit for decay $\Upsilon \rightarrow \mu^{+} \tau^{-}$is lower than $6.0 \times 10^{-6}$ and at $95 \%$ confidence level.

To study the processes $V \rightarrow e^{+} \tau^{-}\left(\mu^{+} \tau^{-}\right)$, the branching ratios versus $\tan \beta_{L}$ are researched. Here, $\tan \beta_{L}=\bar{v}_{L} / v_{L}$, $v_{L}$ and $\bar{v}_{L}$ are the nonzero VEVs of the $S U(2)_{L}$ singlets $\Phi_{L}$ and $\varphi_{L}$. In Fig. 10, using $\tan \beta=6$, $m_{2}=0.5 \mathrm{TeV}, V_{L_{t}}=3 \mathrm{TeV}, A_{L}^{\prime}=0.3 \mathrm{TeV}, S_{m}=1 \mathrm{TeV}$, $M_{L_{f}}^{2}=2 * 10^{5} \mathrm{GeV}^{2}$, and $g_{L}=0.1$, we describe the

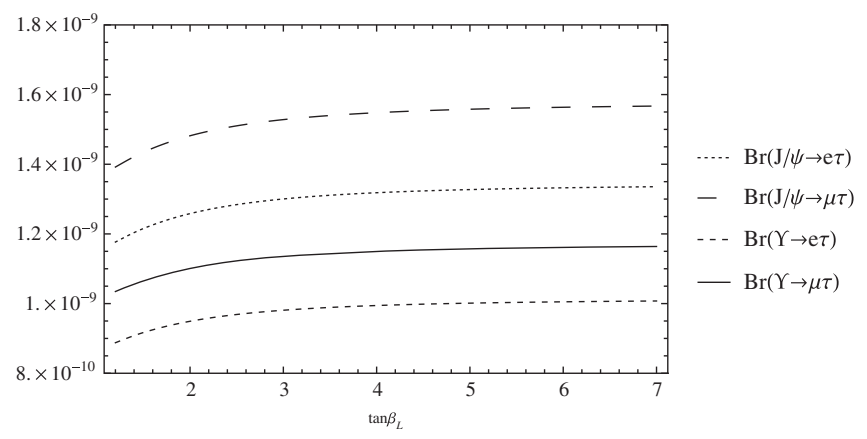

FIG. 10. The branching ratios for $\operatorname{Br}\left(V \rightarrow e^{+} \tau^{-}\left(\mu^{+} \tau^{-}\right)\right) \times$ $(V \in\{J / \Psi, \Upsilon\})$ changing with $\tan \beta_{L}$ are given out.

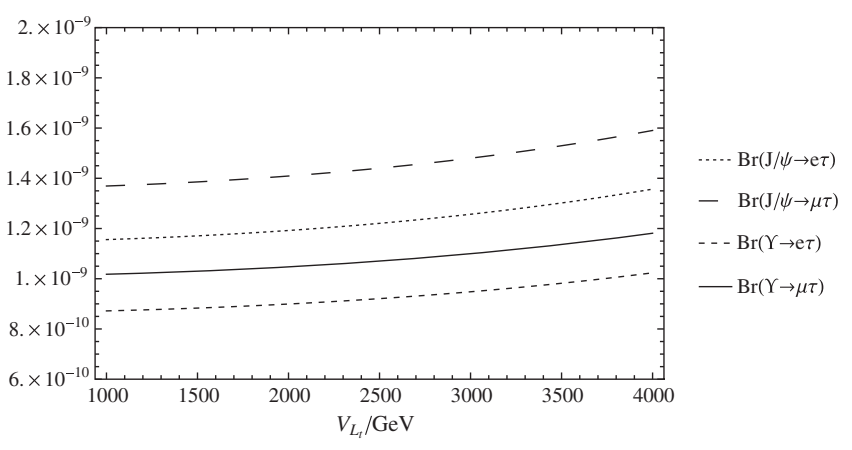

FIG. 11. For $\operatorname{Br}\left(V \rightarrow e^{+} \tau^{-}\left(\mu^{+} \tau^{-}\right)\right), V \in\{J / \Psi, \Upsilon\}$, the results varying with $V_{L_{t}}$ are plotted.

numerical results with the parameter $\tan \beta_{L}$. Obviously, the parameter $\tan \beta_{L}$ has tiny effects on our numerical analysis. When the decay processes have the same initial state, for example, $J / \Psi \rightarrow e^{+} \tau^{-}$and $J / \Psi \rightarrow \mu^{+} \tau^{-}$, the numerical results for both processes are almost the same, and the figure of $J / \Psi \rightarrow e^{+} \tau^{-}$is under that of $J / \Psi \rightarrow \mu^{+} \tau^{-}$. The processes $\Upsilon \rightarrow e^{+} \tau^{-}\left(\mu^{+} \tau^{-}\right)$have the same characters as that of $J / \Psi \rightarrow e^{+} \tau^{-}\left(\mu^{+} \tau^{-}\right)$.

Compared with MSSM, $V_{L_{t}}$ is also a new parameter, which presents in the mass matrices of the slepton, sneutrino, and lepton neutralino. With $V_{L_{t}}=\sqrt{v_{L}^{2}+\bar{v}_{L}^{2}}$, $m_{Z_{L}}=2 g_{L} V_{L_{t}}$ is the mass of neutral $U(1)_{L}$ gauge boson $Z_{L}$. In Fig. 11 , the branching ratios for $V \rightarrow e^{+} \tau^{-}\left(\mu^{+} \tau^{-}\right)$ changing with $V_{L_{t}}$ are discussed, where $\tan \beta=6$, $m_{2}=0.5 \mathrm{TeV}, \quad g_{L}=0.1, \quad \tan \beta_{L}=2, \quad A_{L}^{\prime}=0.3 \mathrm{TeV}$, $S_{m}=1 \mathrm{TeV}$, and $M_{L_{f}}^{2}=2 * 10^{5} \mathrm{GeV}^{2}$. When the values of $V_{L_{t}}$ change from 1 to $4 \mathrm{TeV}$, each figure keeps an increscent variation trend. However, the numerical results all increase slowly, so the effects from $V_{L_{t}}$ are small.

\section{DISCUSSION AND CONCLUSION}

In the frame of the BLMSSM, we study the CLFV decays for vector mesons $V \rightarrow l_{i}^{ \pm} l_{j}^{\mp}$ with $V \in$ $\left\{\phi, J / \Psi, \Upsilon, \rho^{0}, \omega\right\}$ and $l_{i}, l_{j} \in\{e, \mu, \tau\}$. In this model, new parameters and new contributions are considered to these CLFV processes. For example, with the new introduced parameters $g_{L}, \tan \beta_{L}$, and $V_{L_{t}}$, lepton neutralino $\chi_{L}^{0}$ is discussed in our work, which gives new types of contributions through the lepton neutralino-slepton-lepton coupling. Furthermore, three heavy neutrinos and three new scalar neutrinos are also considered in the BLMSSM. The contributions from neutrinos can be neglected due to the tiny Yukawa $Y_{\nu}$. However, the new scalar neutrinos play very important roles, especially the nondiagonal elements $M_{L_{f}}$ in $\left(m_{\tilde{L}}^{2}\right)_{I J}$, which lead to strong mixing for scalar neutrinos of different generation and enhance the lepton flavor violation.

In our previous work, we study the decay processes $l_{j} \rightarrow$ $l_{i}+\gamma$ and the corresponding effective amplitudes are 
shown as $\mathcal{M}_{\mu \rightarrow e \gamma}=e \epsilon^{\mu} \bar{u}_{i}(p+q)\left[q^{2} \gamma_{\mu}\left(C_{1}^{L} P_{L}+C_{1}^{R} P_{R}\right)+\right.$ $\left.m_{l_{j}} i \sigma_{\mu \nu} q^{\nu}\left(C_{2}^{L} P_{L}+C_{2}^{R} P_{R}\right)\right] u_{j}(p)$. In this work, the effective amplitudes for $V \rightarrow e \mu$ are influenced by that of $\mu \rightarrow e \gamma$. Therefore, we take the same parameter space as $\mu \rightarrow e \gamma$ to study the processes $V \rightarrow l_{i}^{ \pm} l_{j}^{\mp}$, and the corresponding constraints from process $\mu \rightarrow e \gamma$ are also taken into account in this work.

Considering the numerical results discussed in Sec. IV, various parameters affect the CLFV decays. $S_{m}$ and $M_{L_{f}}$ are the sensitive parameters, which are the diagonal and nondiagonal elements in matrices $m_{\tilde{L}}$ and $m_{\tilde{R}}$. The influences from $S_{m}$ and $M_{L_{f}}$ are very remarkable. After discussing the constraints on $l_{j} \rightarrow 3 l_{i}, l_{j} \rightarrow l_{i}+\gamma$ and $\mu-e$ conversion, $\operatorname{Br}(\phi \rightarrow e \mu) \sim 10^{-11}, \operatorname{Br}(J / \Psi(\Upsilon) \rightarrow e \mu) \sim$ $10^{-9}$, and $\operatorname{Br}\left(\rho^{0}(\omega) \rightarrow e \mu\right) \sim 2 \times 10^{-12}$, the decays $J / \Psi(\Upsilon) \rightarrow e \mu$ are much easier than $\phi\left(\rho^{0}, \omega\right) \rightarrow e \mu$ to reach the experimental upper bounds. Similarly, $\operatorname{Br}(J / \Psi \rightarrow$ $e \tau(\mu \tau))$ are at the order of $\left(10^{-10} \sim 10^{-9}\right)$, and $\operatorname{Br}(\Upsilon \rightarrow$ $e \tau(\mu \tau)$ ) can reach $5 \times 10^{-10}$, which are very promising to be observed in near-future experiments.

\section{ACKNOWLEDGMENTS}

This work is supported by the Major Project of National Natural Science Foundation of China (NNSFC) (No. 11535002, No. 11605037, No. 11647120, No. 11275036), the Natural Science Foundation of Hebei province with Grants No. A2016201010 and No. A2016201069, and the Natural Science Fund of Hebei University with Grants No. 2011JQ05 and No. 2012- 242, Hebei Key Lab of Optic-Electronic Information and Materials, the Midwest Universities comprehensive strength promotion project.

\section{APPENDIX THE HADRON MATRIX ELEMENTS, ONE-LOOP INTEGRAL FUNCTIONS AND BLMSSM SUPERELDS}

The corresponding hadron matrix elements we used in our work are encoded as
TABLE I. Superfields in the BLMSSM.

\begin{tabular}{lccccc}
\hline \hline Superfields & $S U(3)_{C}$ & $S U(2)_{L}$ & $U(1)_{Y}$ & $U(1)_{B}$ & $U(1)_{L}$ \\
\hline$\hat{Q}$ & 3 & 2 & $1 / 6$ & $1 / 3$ & 0 \\
$\hat{U}^{c}$ & $\overline{3}$ & 1 & $-2 / 3$ & $-1 / 3$ & 0 \\
$\hat{D}^{c}$ & $\overline{3}$ & 1 & $1 / 3$ & $-1 / 3$ & 0 \\
$\hat{L}$ & 1 & 2 & $-1 / 2$ & 0 & 1 \\
$\hat{E}^{c}$ & 1 & 1 & 1 & 0 & -1 \\
$\hat{N}^{c}$ & 1 & 1 & 0 & 0 & -1 \\
$\hat{Q}_{4}$ & 3 & 2 & $1 / 6$ & $B_{4}$ & 0 \\
$\hat{U}_{4}^{c}$ & $\overline{3}$ & 1 & $-2 / 3$ & $-B_{4}$ & 0 \\
$\hat{D}_{4}^{c}$ & $\overline{3}$ & 1 & $1 / 3$ & $-B_{4}$ & 0 \\
$\hat{L}_{4}$ & 1 & 2 & $-1 / 2$ & 0 & $L_{4}$ \\
$\hat{E}_{4}^{c}$ & 1 & 1 & 1 & 0 & $-L_{4}$ \\
$\hat{N}_{4}^{c}$ & 1 & 1 & 0 & 0 & $-L_{4}$ \\
$\hat{Q}_{5}^{c}$ & $\overline{3}$ & 2 & $-1 / 6$ & $-1-B_{4}$ & 0 \\
$\hat{U}_{5}$ & 3 & 1 & $2 / 3$ & $1+B_{4}$ & 0 \\
$\hat{D}_{5}$ & 3 & 1 & $-1 / 3$ & $1+B_{4}$ & 0 \\
$\hat{L}_{5}^{c}$ & 1 & 2 & $1 / 2$ & 0 & $-3-L_{4}$ \\
$\hat{E}_{5}$ & 1 & 1 & -1 & 0 & $3+L_{4}$ \\
$\hat{N}_{5}$ & 1 & 1 & 0 & 0 & $3+L_{4}$ \\
$\hat{H}_{u}$ & 1 & 2 & $1 / 2$ & 0 & 0 \\
$\hat{H}^{d}$ & 1 & 2 & $-1 / 2$ & 0 & 0 \\
$\hat{\Phi}_{B}$ & 1 & 1 & 0 & 1 & 0 \\
$\hat{\varphi}_{B}$ & 1 & 1 & 0 & -1 & 0 \\
$\hat{\Phi}_{L}$ & 1 & 1 & 0 & 0 & -2 \\
$\hat{\varphi}_{L}$ & 1 & 1 & 0 & 0 & 2 \\
$\hat{X}^{c}$ & 1 & 1 & 0 & $2 / 3+B_{4}$ & 0 \\
$\hat{X}^{\prime}$ & 1 & 1 & 0 & $-2 / 3-B_{4}$ & 0 \\
\hline \hline & & & & & \\
& 1 & $1 / 2$ & 0 & 0 \\
\hline & 1 & 1 & 0 & 0 \\
\hline
\end{tabular}

$$
\begin{aligned}
\left\langle 0\left|\bar{v}_{V}\left(p_{2}\right) \gamma^{\mu} u_{V}\left(p_{1}\right)\right| V(p)\right\rangle & =\frac{f_{V} m_{V}}{2 N_{c}} \varepsilon^{\mu *}(p), \\
\left\langle 0\left|\bar{v}_{V}\left(p_{2}\right) \sigma^{\mu \nu} P_{L} u_{V}\left(p_{1}\right)\right| V(p)\right\rangle & =-\frac{f_{V} \epsilon^{\mu \nu p \varepsilon^{*}(p)}+i f_{V}\left(p^{\mu} \varepsilon^{\nu *}(p)-p^{\nu} \varepsilon^{\mu *}(p)\right)}{2 N_{c}}, \\
\left\langle 0\left|\bar{v}_{V}\left(p_{2}\right) \sigma^{\mu \nu} P_{R} u_{V}\left(p_{1}\right)\right| V(p)\right\rangle & =\frac{f_{V} \epsilon^{\mu \nu p \varepsilon^{*}(p)}-i f_{V}\left(p^{\mu} \varepsilon^{\nu *}(p)-p^{\nu} \varepsilon^{\mu *}(p)\right)}{2 N_{c}}, \\
\left\langle 0\left|\bar{v}_{V}\left(p_{2}\right) u_{V}\left(p_{1}\right)\right| V(p)\right\rangle & =\left\langle 0\left|\bar{v}_{V}\left(p_{2}\right) \gamma_{5} u_{V}\left(p_{1}\right)\right| V(p)\right\rangle=\left\langle 0\left|\bar{v}_{V}\left(p_{2}\right) \gamma^{\mu} \gamma_{5} u_{V}\left(p_{1}\right)\right| V(p)\right\rangle=0 .
\end{aligned}
$$

We find that $\left\langle 0\left|\bar{v}_{V}\left(p_{2}\right) \gamma^{\mu} u_{V}\left(p_{1}\right)\right| V(p)\right\rangle$ plays the dominant contribution in our numerical results. We can almost ignore the effects from the other hadron matrix elements in Eq. (36). 
In this section, we give out the corresponding one-loop integral functions, which read as

$$
\begin{aligned}
& I_{1}\left(x_{1}, x_{2}\right)=\frac{1}{16 \pi^{2}}\left[-\left(\triangle+1+\ln x_{\mu}\right)+\frac{x_{2} \ln x_{2}-x_{1} \ln x_{1}}{\left(x_{2}-x_{1}\right)}\right] \text {, } \\
& I_{2}\left(x_{1}, x_{2}\right)=\frac{1}{32 \pi^{2}}\left[\frac{3+2 \ln x_{2}}{\left(x_{2}-x_{1}\right)}-\frac{2 x_{2}+4 x_{2} \ln x_{2}}{\left(x_{2}-x_{1}\right)^{2}}+\frac{2 x_{2}^{2} \ln x_{2}-2 x_{1}^{2} \ln x_{1}}{\left(x_{2}-x_{1}\right)^{3}}\right] \text {, } \\
& I_{3}\left(x_{1}, x_{2}\right)=\frac{1}{16 \pi^{2}}\left[\frac{1+\ln x_{2}}{\left(x_{2}-x_{1}\right)}+\frac{x_{1} \ln x_{1}-x_{2} \ln x_{2}}{\left(x_{2}-x_{1}\right)^{2}}\right] \text {, } \\
& I_{4}\left(x_{1}, x_{2}\right)=\frac{1}{96 \pi^{2}}\left[\frac{11+6 \ln x_{2}}{\left(x_{2}-x_{1}\right)}-\frac{15 x_{2}+18 x_{2} \ln x_{2}}{\left(x_{2}-x_{1}\right)^{2}}+\frac{6 x_{2}^{2}+18 x_{2}^{2} \ln x_{2}}{\left(x_{2}-x_{1}\right)^{3}}+\frac{6 x_{1}^{3} \ln x_{1}-6 x_{2}^{3} \ln x_{2}}{\left(x_{2}-x_{1}\right)^{4}}\right] \text {, } \\
& I_{5}\left(x_{1}, x_{2}\right)=\frac{1}{16 \pi^{2}}\left[\left(\Delta+1+\ln x_{\mu}\right)+\frac{x_{2}+2 x_{2} \ln x_{2}}{\left(x_{1}-x_{2}\right)}+\frac{x_{2}^{2} \ln x_{2}-x_{1}^{2} \ln x_{1}}{\left(x_{2}-x_{1}\right)^{2}}\right] \text {, } \\
& G_{1}\left(x_{1}, x_{2}, x_{3}\right)=\frac{1}{16 \pi^{2}}\left[\frac{x_{1} \ln x_{1}}{\left(x_{1}-x_{2}\right)\left(x_{1}-x_{3}\right)}+\frac{x_{2} \ln x_{2}}{\left(x_{2}-x_{1}\right)\left(x_{2}-x_{3}\right)}+\frac{x_{3} \ln x_{3}}{\left(x_{3}-x_{1}\right)\left(x_{3}-x_{2}\right)}\right], \\
& G_{2}\left(x_{1}, x_{2}, x_{3}\right)=\frac{1}{16 \pi^{2}}\left[-\left(\triangle+1+\ln x_{\mu}\right)+\frac{x_{1}^{2} \ln x_{1}}{\left(x_{1}-x_{2}\right)\left(x_{1}-x_{3}\right)}\right. \\
& \left.+\frac{x_{2}^{2} \ln x_{2}}{\left(x_{2}-x_{1}\right)\left(x_{2}-x_{3}\right)}+\frac{x_{3}^{2} \ln x_{3}}{\left(x_{3}-x_{1}\right)\left(x_{3}-x_{2}\right)}\right] \text {, } \\
& J_{1}\left(x_{1}, x_{2}, x_{3}, x_{4}\right)=\frac{1}{16 \pi^{2}}\left[\frac{x_{1}^{2} \ln x_{1}}{\left(x_{1}-x_{2}\right)\left(x_{1}-x_{3}\right)\left(x_{1}-x_{4}\right)}+\frac{x_{2}^{2} \ln x_{2}}{\left(x_{2}-x_{1}\right)\left(x_{2}-x_{3}\right)\left(x_{2}-x_{4}\right)}\right. \\
& \left.+\frac{x_{3}^{2} \ln x_{3}}{\left(x_{3}-x_{1}\right)\left(x_{3}-x_{2}\right)\left(x_{3}-x_{4}\right)}+\frac{x_{4}^{2} \ln x_{4}}{\left(x_{4}-x_{1}\right)\left(x_{4}-x_{2}\right)\left(x_{4}-x_{3}\right)}\right] \text {, } \\
& J_{2}\left(x_{1}, x_{2}, x_{3}, x_{4}\right)=\frac{1}{16 \pi^{2}}\left[\frac{x_{1} \ln x_{1}}{\left(x_{1}-x_{2}\right)\left(x_{1}-x_{3}\right)\left(x_{1}-x_{4}\right)}+\frac{x_{2} \ln x_{2}}{\left(x_{2}-x_{1}\right)\left(x_{2}-x_{3}\right)\left(x_{2}-x_{4}\right)}\right. \\
& \left.+\frac{x_{3} \ln x_{3}}{\left(x_{3}-x_{1}\right)\left(x_{3}-x_{2}\right)\left(x_{3}-x_{4}\right)}+\frac{x_{4} \ln x_{4}}{\left(x_{4}-x_{1}\right)\left(x_{4}-x_{2}\right)\left(x_{4}-x_{3}\right)}\right] \text {, }
\end{aligned}
$$

with $\Delta=\frac{1}{\epsilon}-r_{\epsilon}+\ln 4 \pi$.

We also describe the corresponding superfields presented in the BLMSSM model, which are shown in Table I.

[1] K. Abe et al. (T2K Collab), Phys. Rev. Lett. 107, 041801 (2011).

[2] P. Adamson et al. (MINOS Collaboration), Phys. Rev. Lett. 107, 181802 (2011).

[3] Y. Abe et al. (DOUBLE-CHOOZ Collaboration), Phys. Rev. Lett. 108, 131801 (2012).

[4] F. An et al. (DAYA-BAY Collaboration), Phys. Rev. Lett. 108, 171803 (2012).

[5] J.Ahn et al. (RENO Collaboration), Phys. Rev. Lett. 108, 191802 (2012).

[6] P. Ghosh and S. Roy, J. High Energy Phys. 04 (2009) 069.

[7] M. C. Gonzalez-Garcia and M. Maltoni, Phys. Rep. 460, 1 (2008).

[8] M. C. Gonzalez-Garcia, M. Maltoni, J. Salvado, and T. Schwetz, J. High Energy Phys. 12 (2012) 123.
[9] I. Girardi, S. T. Petcov, and A. V. Titov, Nucl. Phys. B894, 733 (2015).

[10] A. Abada, D. Das, A. Vicente, and C. Weiland, J. High Energy Phys. 09 (2012) 015.

[11] A. Ilakovac, A. Pilaftsis, and L. Popov, Phys. Rev. D 87, 053014 (2013).

[12] A. Abada, V. De Romeri, and A. M. Teixeira, J. High Energy Phys. 02 (2016) 083.

[13] J. C. Pati and A. Salam, Phys. Rev. D 10, 275 (1974).

[14] H. Georgi and S. L. Glashow, Phys. Rev. Lett. 32, 438 (1974).

[15] H. P. Nilles, Phys. Rep. 110, 1 (1984).

[16] H. E. Haber and G. L. Kane, Phys. Rep., 117, 75 (1985).

[17] C. H. Chang and T. F. Feng, Eur. Phys. J. C 12, 137 (2000).

[18] R. N. Mohapatra and J. C. Pati, Phys. Rev. D 11, 566 (1975). 
[19] G. Senjanovic and R. N. Mohapatra, Phys. Rev. D 12, 1502 (1975).

[20] A. Leike, Phys. Rep. 317, 143 (1999).

[21] P. Langacker, Rev. Mod. Phys. 81, 1199 (2009).

[22] K. S. Sun, T. F. Feng, T. J. Gao, and S.-M. Zhao, Nucl. Phys. B865, 486 (2012).

[23] K. S. Sun, T. F. Feng, L. N. Kou, F. Sun, T.-J. Gao, and H.-B. Zhang, Mod. Phys. Lett. A 27, 1250172 (2012).

[24] S. M. Zhao, T. F. Feng, H. B. Zhang, X.-J. Zhan, Y.-J. Zhang, and B. Yan, Phys. Rev. D 92, 115016 (2015).

[25] S. Nussinov, R. D. Peccei, and X. M. Zhang, Phys. Rev. D 63, 016003 (2000).

[26] A. Faessler, Th. Gutsche, S. Kovalenko, V. E. Lyubovitskij, I. Schmidt, and F. Šimkovic, Phys. Lett. B 590, 57 (2004).

[27] A. Faessler, Th. Gutsche, S. Kovalenko, V. E. Lyubovitskij, I. Schmidt, and F. Šimkovic, Phys. Rev. D 70, 055008 (2004).

[28] T. Gutsche, J. Helo, S. Kovalenko, and V. Lyubovitskij, Phys. Rev. D 81, 037702 (2010).

[29] C. Patrignani et al. (Particle Data Group), Chin. Phys. C 40, 100001 (2016).

[30] J. Rosiek, Phys. Rev. D 41, 3464 (1990).

[31] T. F. Feng and X. Y. Yang, Nucl. Phys. B814, 101 (2009).

[32] P. F. Perez and M. B. Wise, Phys. Rev. D 82, 011901 (2010).

[33] P.F. Perez and M.B. Wise, Phys. Rev. D 84, 055015 (2011).

[34] P. F. Perez and M. B. Wise, J. High Energy Phys. 08 (2011) 068.

[35] J. M. Amold, P. F. Perez, B. Formal, and S. Spinner, Phys. Rev. D 85, 115024 (2012).

[36] T. R. Dulaney, P. F. Perez, and M. B. Wise, Phys. Rev. D 83, 023520 (2011).

[37] P. F. Perez, Phys. Lett. B 711, 353 (2012).
[38] T.-F. Feng, S.-M. Zhao, H.-B. Zhang, Y.-J. Zhang, and Y.-L. Yan, Nucl. Phys. B871, 223 (2013).

[39] S.-M. Zhao, T.-F. Feng, B. Yan, H.-B. Zhang, Y.-J. Zhang, B. Chen, and X.-Q. Li, J. High Energy Phys. 10 (2013) 020.

[40] S.-M. Zhao, T.-F. Feng, H.-B. Zhang, B. Yan, and X.-J. Zhan, J. High Energy Phys. 11 (2014) 119.

[41] M. A. Shifman, A. I. Vainshtein, and V. I. Zakharov, Nucl. Phys. B147, 385 (1979).

[42] V. L. Chernyak and A. R. Zhitnitsky, Phys. Rep. 112, 173 (1984).

[43] I. I. Balitsky, V. M. Braum, and A. V. Kolesnichenko, Nucl. Phys. B312, 509 (1989).

[44] P. Ball and V. M. Braun, Phys. Rev. D 54, 2182 (1996); P. Ball, J. High Energy Phys. 09 (1998) 005.

[45] G. Li, T. Li, X.-Q. Li, W.-G. Ma, and S.-M. Zhao, Nucl. Phys. B727, 301 (2005); T. Li, S. M. Zhao, and X. Q. Li, Nucl. Phys. A828, 125 (2009).

[46] M. Beneke, G. Buchalla, M. Neubert, and C. T. Sachrajda, Nucl. Phys. B591, 313 (2000).

[47] F. Fierz, Z. Phys. 104, 553 (1937).

[48] A. Salam and J. Strather, Fortschr. Phys. 26, 57 (1978).

[49] P. Fayet and S.Ferrara, Phys. Rep. 32, 249 (1977).

[50] ATLAS Collaboration, Phys. Lett. B 716, 1 (2012).

[51] CMS Collaboration, Phys. Lett. B 716, 30 (2012).

[52] CMS Collaboration, J. High Energy Phys. 06 (2013) 081.

[53] M. G. Garcia, M. Maltoni, J.Salvado, and T. Schwetz, J. High Energy Phys. 12 (2012) 123.

[54] D. V. Ferero, M. Trtola, and J. W. F. Valle, Phys. Rev. D 86, 073012 (2012).

[55] X.-X. Dong, S.-M. Zhao, X.-J. Zhan, Z.-J. Yang, H.-B. Zhang, and T.-F. Feng, Chin. Phys. C 41, 073103 (2017).

[56] S.-M. Zhao, T.-F. Feng, X.-J. Zhan, H.-B. Zhang, and B. Yan, J. High Energy Phys. 07 (2015) 124. 